\title{
First-principles calculations of the phase stability of $\mathrm{TiO}_{2}$
}

\author{
Joseph Muscat, ${ }^{1}$ Varghese Swamy, ${ }^{2}$ and Nicholas M. Harrison ${ }^{3}$ \\ ${ }^{1}$ CSIRO Minerals, Box 312, Clayton South, Victoria 3169, Australia \\ ${ }^{2}$ CRCT, Ecole Polytechnique de Montreal, C.P. 6079, succursale Centre-Ville, Montreal, Quebec H3C 3A7, Canada \\ ${ }^{3}$ CCLRC Daresbury Laboratory, Daresbury, Warrington, WA4 4AD, United Kingdom \\ and Department of Chemistry, Imperial College of Science and Technology, \\ London, SW7 2AY, United Kingdom
}

(Received 4 January 2002; revised manuscript received 28 March 2002; published 11 June 2002)

\begin{abstract}
First-principles calculations of the crystal structures, bulk moduli, and relative stabilities of seven known and hypothetical $\mathrm{TiO}_{2}$ polymorphs (anatase, rutile, columbite, baddeleyite, cotunnite, pyrite, and fluorite structures) have been carried out with the all-electron linear combination of atomic orbitals (LCAO) and pseudopotential planewave (PW) methods. The anatase versus rutile relative phase stability at $0 \mathrm{~K}$ and zero pressure has been investigated using high-quality basis sets and carefully controlled computational parameters. From the optimal crystal structures obtained with the Hartree-Fock theory at various pressures, the bulk modulus and phase transition pressures of various high-pressure polymorphs have been derived at the athermal limit. In most cases, the calculated unit cell data agree to within $2 \%$ of the corresponding experimental determination. Complete predicted structural data (unit cell constants and fractional atomic coordinates) are presented for the baddeleyite and pyrite forms. The calculated bulk moduli are within $10 \%$ of the most reliable experimental results. Both the all-electron LCAO and pseudopotential PW methods predict anatase to be more stable than rutile at $0 \mathrm{~K}$ and ambient pressure. The computed anatase-columbite, rutile-columbite, columbite-baddeleyite, and baddeleyite-cotunnite phase transitions appear in the same order as observed in experiments, and the transition pressures agree semiquantitatively with those measured. The pyrite and fluorite structures are predicted to be less stable than other polymorphs at pressures below $70 \mathrm{GPa}$ in agreement with experiments. Finally, the elastic properties, compressibilities and phase transformations of the various polymorphs are discussed in terms of simple models based on the behavior of the constituent Ti-O polyhedra under compression.
\end{abstract}

DOI: 10.1103/PhysRevB.65.224112

PACS number(s): 61.50.Ah, 61.50.Ks, 81.30.Hd, 61.66.Fn

\section{INTRODUCTION}

$\mathrm{TiO}_{2}$ occurs in Nature in three different forms which, in order of abundance, are rutile, anatase, and brookite. The rutile form is widely used as a white pigment and opacifier. The anatase phase finds applications in, among others, photocatalysts and nanostructured solar cells. ${ }^{1-4}$ The rutile and anatase phases have been widely studied in recent years. The rarer mineral brookite is not used commercially; this, coupled with its relatively complicated structure, has led to few studies of its properties.

At elevated pressures $\mathrm{TiO}_{2}$ has a rich phase diagram with a series of structural phase transformations. The structure and stability of the high-pressure phases of $\mathrm{TiO}_{2}$ are of particular interest in Earth sciences, for these phases are an accessible analog of minerals in the Earth's mantle. Rutile $\mathrm{TiO}_{2}$ is particularly attractive in this context because it is expected to undergo a sequence of phase transformations with increasing pressure similar to that experienced by stishovite $\mathrm{SiO}_{2}$ in the Earth's mantle, but at more readily accessible pressures. Great interest in the high-pressure phases has also been stimulated by the recent discovery of the ultrahard $\mathrm{TiO}_{2}$ cotunnite phase. This phase can be stabilized in diamond-anvil cell experiments at about $60 \mathrm{GPa}$, and is the hardest known oxide material $;{ }^{5}$ its discovery has stimulated much interest in potential applications of pressure-stabilized phases.

There have been a number of attempts to determine the relative stability of rutile and anatase using calorimetric methods. ${ }^{6-10}$ However, the enthalpy change of the anatase to rutile transformation remains somewhat controversial, with room-temperature experiments yielding values ranging from $\Delta H_{298}=-11.7 \mathrm{~kJ} / \mathrm{mol}$ (Ref. 7) to $+0.42 \mathrm{~kJ} / \mathrm{mol}$ (Ref. 10). A number of first-principles studies have been performed to address this issue in recent years. Whereas all the studies reproduce observed crystal structures well, the predicted phase stability is found to be sensitive to the treatment of electronic exchange and correlation ${ }^{11-13}$ and on the numerical details of the calculations. ${ }^{14}$

High pressure $\mathrm{x}$-ray-diffraction ${ }^{15,16,21,23-25}$ and Raman spectroscopy ${ }^{17-20,22}$ studies have revealed that rutile and anatase transform to a columbite structure at high pressure. The pressure at which this transformation occurs depends on the starting material: anatase transforms to columbite at 4-8 $\mathrm{GPa},{ }^{20-22}$ whereas rutile undergoes a sluggish transformation at about $10 \mathrm{GPa}{ }^{17,19,20}$ Furthermore, the phase transformation from anatase to the columbite structure is dependent on whether or not a single crystal sample is used ${ }^{25}$ and on the temperature at which the experiment is performed. ${ }^{24}$ In recent $\mathrm{x}$-ray-diffraction studies it has been reported that, at room temperature, the columbite phase is only formed at about $7 \mathrm{GPa}$ during decompression from a higher pressure phase. ${ }^{23-25}$ However, in Raman studies the transformation of rutile and anatase directly to columbite has been observed at 5 GPa. ${ }^{17-20,22,84}$ The columbite phase formed from either rutile or anatase is metastable upon decompression, and can 
also be obtained at ambient pressures by acid dissolution of $\mathrm{Ti}_{3} \mathrm{O}_{5} \cdot{ }^{27}$

Columbite-structured $\mathrm{TiO}_{2}$ transforms to a baddeleyite structured phase between 12 and $17 \mathrm{GPa} .{ }^{19,22,23,26}$ This structure is significantly more complex than columbite, and is harder to obtain and characterize experimentally because of the difficulties involved in applying higher pressures and in quenching the high-pressure phase. This complexity also means that it is computationally demanding to study theoretically; to our knowledge, there has only been one previous $a b$ initio determination of the lattice vectors and internal coordinates of this phase. ${ }^{13}$

The situation regarding post-baddeleyite transition(s) has been less clear until very recently. A number of observations suggested a transformation to a cubic phase at pressures of about $60 \mathrm{GPa}$ but with insufficient data available to fully determine the structure. ${ }^{22,28,29}$ Several metal oxides, which are rutile structured at $0 \mathrm{GPa}$, are known to transform to fluorite structured phases at high pressure, and, on this basis, it has been postulated that this phase adopts a fluorite structure. $^{22,28,29}$ Later studies including Rietveld refinement of x-ray-diffraction data from three rutile-structured oxides $\left(\mathrm{SnO}_{2}, \mathrm{PbO}_{2}\right.$, and $\mathrm{RuO}_{2}$ ) revealed that the high-pressure phase in these systems actually adopts a pyrite structure. ${ }^{39}$ Several theoretical studies have been performed to assist in the identification of these phases. In particular, the cubic $\mathrm{CaF}_{2}$ (fluorite) structure ${ }^{30}$ and a modification of this form which is isostructural with $\mathrm{FeS}_{2}$ (pyrite) have been proposed as candidate high-pressure phases. ${ }^{31}$

Unfortunately, confirming the existence of these predicted structures by performing experiments at such high pressures poses many technical difficulties ${ }^{22}$ which had, until recently, precluded the detailed determination of the post-baddeleyite phase. However, a recent study which included Reitveld refinement of phases synthesised at pressures up to $80 \mathrm{GPa}$ revealed a transition from a baddeleyite to a cotunnite $\left(\mathrm{PbCl}_{2}\right)$ structure at $60 \mathrm{GPa}$, which was concurrently verified by means of first-principles and lattice-dynamics calculations. ${ }^{5}$

Calculations of carefully controlled numerical precision have a key role to play in determining the stability and structure of materials under conditions that are difficult to reproduce in the laboratory. A detailed description of the rich phase diagram, including phases with sixfold, sevenfold, eightfold, and ninefold Ti-O coordinations is a challenge for any theoretical treatment of the bonding and energetics of $\mathrm{TiO}_{2}$. Much progress in understanding the bulk and defect chemistry of $\mathrm{TiO}_{2}$ has been made with empirical forcefield modeling. ${ }^{32}$ However, such models often have limited predictive power, failing when applied to systems not taken into account in the original parametrization of the model. Despite recent efforts to develop force fields that can treat different coordination environments, it has been found that their performance varies from phase to phase depending on the property being computed, and these models give poor results for some phases. ${ }^{33}$ First-principles calculations provide an unbiased and thus predictive approach to the modeling of phases for which experimental data are not available. However, in applying first-principles techniques to complex materials, electron exchange and correlation interactions must be approximated and a number of numerical approximations adopted.

In this paper the results of high-quality first-principles calculations of the structure and phase stability of $\mathrm{TiO}_{2}$ over a wide range of pressures are presented. For known polymorphs the computed data are compared to that observed in order to establish the validity of predictions for higher-pressure phases. The stability of some key results with respect to the treatment of exchange and correlation and to numerical approximations is explored. The delicate relative stability of rutile and anatase at ambient pressure is studied using both high quality, all-electron, linear combination of atomic orbital (LCAO) and pseudopotential, plane-wave (PW) methods. A determination of the structures of the high-pressure phases baddeleyite and pyrite is presented. The stability of the cotunnite phase relative to the pyrite and fluorite phases below $60 \mathrm{GPa}$ is confirmed. Finally, these calculations offer valuable insights into the nature of the phase transformations and the crucial link between the structure of a material on the atomic scale and its macroscopic properties.

The paper is organized as follows. In Sec. II a brief description of structures of the different phases considered in this paper is given. Section III contains details of the computational methods used. Computed data including the phase stability, structure, and elastic properties of rutile and anatase are given in Sec. IV A, and data regarding the high-pressure phases is given in Sec. IV B. These results are discussed in Sec. V and conclusions drawn in section VI.

\section{POLYMORPHS OF $\mathrm{TiO}_{2}$}

As an understanding of the crystal structure and bonding of $\mathrm{TiO}_{2}$ is important to the work presented in this paper, we describe the structure of the various polymorphs in some detail.

\section{A. Rutile}

The vast majority of studies of the bulk and surface properties of $\mathrm{TiO}_{2}$ have been of the rutile phase. The rutile structure, illustrated in Fig. 1, belongs to the $P 4_{2} / \mathrm{mnm}$ tetragonal space group. The unit cell is defined by the lattice vectors a and $\mathbf{c}$ and contains two $\mathrm{TiO}_{2}$ units with $\mathrm{Ti}$ ions at $(0,0,0)$ and $\left(\frac{1}{2}, \frac{1}{2}, \frac{1}{2}\right)$ and $\mathrm{O}$ ions at $\pm(u, u, 0)$ and $\pm\left(u+\frac{1}{2}, \frac{1}{2}-u, \frac{1}{2}\right)$. The unit-cell parameters have been determined and subsequently verified several times using $\mathrm{x}-\mathrm{ray}^{34,35}$ and neutron diffraction, ${ }^{36,37}$ and are found to be $\mathbf{a}=4.587 \AA$, $\mathbf{c}$ $=2.954 \AA$, and $\mathbf{u}=0.305$ at $15 \mathrm{~K}$ (Ref. 37) (see Table I). Each $\mathrm{Ti}$ ion is octahedrally coordinated to six $\mathrm{O}$ ions. The $\mathrm{TiO}_{2}$ octahedron is distorted, with the apical Ti-O bond length $(1.98 \AA)$ being slightly longer than the equatorial Ti-O bond length $(1.95 \AA)$. The four equatorial $\mathrm{O}$ ions are coplanar occupying a rectangular arrangement with the long edge $(2.954 \AA)$ along the c direction and the short edge $(2.53 \AA)$ lying diagonally across the plane defined by the a direction. The $\mathrm{TiO}_{6}$ octahedra form chains that share edges along the $\mathbf{c}$ direction and share vertices in the a-b plane (see Fig. 6 in Sec. V). 


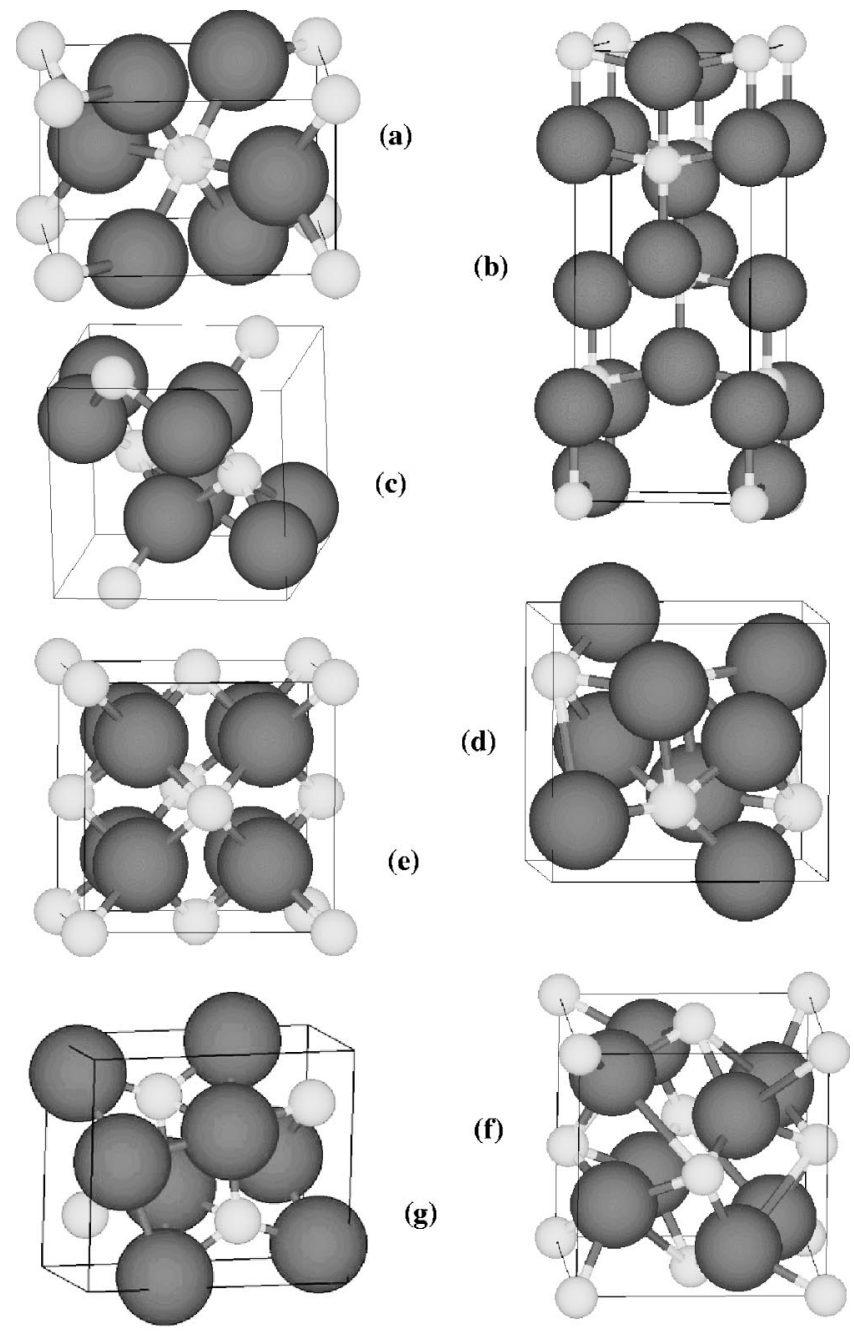

FIG. 1. The phases of $\mathrm{TiO}_{2}$ studied here: rutile (a), anatase (b), columbite (c), baddeleyite (d), fluorite (e), pyrite (f), and cotunnite (g). Large spheres represent the $\mathrm{O}$ ions, small spheres the Ti ions.

\section{B. Anatase}

The anatase structure, shown in Fig. 1, is characterized by the tetragonal space group I4/amd. The unit cell contains two $\mathrm{TiO}_{2}$ units with $\mathrm{Ti}$ ions at $(0,0,0)$ and $\left(0, \frac{1}{2}, \frac{1}{4}\right)$ and $\mathrm{O}$ ions at $(0,0, u),(0,0, \bar{u}),\left(0, \frac{1}{2}, u+\frac{1}{4}\right)$ and $\left(0, \frac{1}{2}, \frac{1}{2}-u\right)$. As with rutile, $\mathbf{a}, \mathbf{c}$, and $\mathbf{u}$ have been measured several times using both $\mathrm{x}$-ray ${ }^{34,35}$ and neutron diffraction ${ }^{36,37}$ to be $3.782 \AA$, $9.502 \AA$, and 0.208 , respectively, at about $300 \mathrm{~K}$ (see Table I). Each $\mathrm{Ti}$ ion is octahedrally coordinated to six $\mathrm{O}$ ions. The $\mathrm{Ti}-\mathrm{O}$ octahedron is not regular and the Ti-O bond distances are similar to those in rutile (1.98 and $1.93 \AA$ for the long and short Ti-O bond lengths respectively). The octahedra form zigzag chains along the $\mathbf{a}$ and $\mathbf{b}$ directions with each octahedron sharing four edges (see Fig. 7 in Sec. V).

\section{Columbite}

The columbite structure, presented in Fig. 1, has an orthorhombic unit cell with the $P b c n$ space group and lattice vectors $\mathbf{a}=4.541 \AA \mathbf{b}=5.493 \AA$, and $\mathbf{c}=4.906 \AA$. There are four $\mathrm{TiO}_{2}$ units per cell with $\mathrm{Ti}$ ions at $\pm\left(0, u, \frac{1}{2}\right)$ and $\pm\left(\frac{1}{2}, u+\frac{1}{2}, \frac{1}{4}\right)$ and $\mathrm{O}$ ions at $\pm(x, y, z), \pm\left(\frac{1}{2}-x, \frac{1}{2}-y, z+\frac{1}{2}\right)$, $\pm\left(x+\frac{1}{2}, \frac{1}{2}-y, \bar{z}\right)$, and $\left(\bar{x}, y, \frac{1}{2}-z\right)$. The internal fractional coordinates $(\mathbf{u}, \mathbf{x}, \mathbf{y}$, and $\mathbf{z})$ have been determined in highpressure X-ray-diffraction studies to be $0.171,0.286,0.376$, and 0.412 , respectively. ${ }^{38}$ As with rutile and anatase, each Ti ion is octahedrally coordinated to six $\mathrm{O}$ ions. The octahedron is distorted with the cation displaced from the center of the octahedron. The Ti-O bond distances range from 1.91 to 2.05 $\AA$, with a mean bond length similar to that of rutile. The octahedra form planar chains sharing edges in a zigzag arrangement along the c direction (see Fig. 8 in Sec. V).

\section{Baddeleyite}

The baddeleyite phase is formed under compression to about $20 \mathrm{GPa}^{26}$ The unit cell of this phase is displayed in Fig. 1. Baddeleyite is often described as an intermediate structure between rutile and fluorite, and is adopted by several other materials including $\mathrm{ZrO}_{2}$. The unit cell is monoclinic (space group $P 2_{1} / c$ ) containing four $\mathrm{TiO}_{2}$ units with $\mathrm{Ti}$ and $\mathrm{O}$ ions at $\pm\left(x, y, z ; \bar{x}, y+\frac{1}{2}, \frac{1}{2}-z\right)$. The lattice vectors have been measured in $\mathrm{x}$-ray studies and are (extrapolated to $0 \mathrm{GPa}) \mathbf{a}=4.662 \AA, \mathbf{b}=4.969 \AA, \mathbf{c}=4.911 \AA$, and $\beta$ $=99.4^{\circ} .^{23}$ The internal coordinates $x, y$, and $z$ of the ions in this structure have not previously been determined, to our knowledge. Each $\mathrm{Ti}$ ion is coordinated to seven $\mathrm{O}$ ions, and the oxygen ions form alternating threefold- and fourfoldcoordinated layers.

\section{E. Fluorite}

Very high-pressure experiments have suggested that $\mathrm{TiO}_{2}$ transforms to a cubic structure at pressures in excess of about $60 \mathrm{GPa}^{22,28,29}$ It has been suggested that this phase has the fluorite $\left(\mathrm{CaF}_{2}\right)$ structure characterized by the $F m 3 m$ space group. Structural data for this phase have yet to be determined.

The fluorite structure has one formula unit per primitive cell with the cation occupying the $(0,0,0)$ position while the anions are at $\pm\left(\frac{1}{4}, \frac{1}{4}, \frac{1}{4}\right)$, as shown in Fig. 1. Each Ti ion is coordinated to eight $\mathrm{O}$ ions, and hence each $\mathrm{O}$ ion is tetrahedrally coordinated to Ti ions.

\section{F. Pyrite}

$\mathrm{SnO}_{2}, \mathrm{RuO}_{2}$, and $\mathrm{ZrO}_{2}$, which all have a rutile structure at $0 \mathrm{GPa}$, have also been observed to transform to a cubic structure at high pressures. ${ }^{39}$ This structure was assumed to be fluorite, but recent Rietveld refinement from x-raydiffraction data has revealed that the cubic phase is actually a modified or distorted fluorite structure isostructural to pyrite $\mathrm{FeS}_{2}$ (space group $P a \overline{3}$ ). As the $P a \overline{3}$ space group is a subgroup of $F m 3 m$, a continuous phase transformation from one to the other is possible. The main difference between this structure and the fluorite structure is that the $\mathrm{O}$ ions are displaced from $\pm\left(\frac{1}{4}, \frac{1}{4}, \frac{1}{4}\right)$ to about $\pm(0.34,0.34,0.34)$ (see Fig. 1). This leads to each Ti ion having an inner shell of six $O$ 
TABLE I. A selection of previous theoretical and experimental structural parameters for rutile and anatase (in $\AA$ ). The abbreviations describing the methods used in the theoretical studies are given in Sec. III. The LCAO-HF (Refs. 11,57,58,63 and 65) and LCAO-LDA (Ref. 63) calculations are performed using the CRYSTAL package. Numbers in parentheses indicate the percent deviation from low-temperature neutrondiffraction experiments.

\begin{tabular}{|c|c|c|c|c|c|c|}
\hline \multirow{2}{*}{ Method } & \multicolumn{2}{|c|}{$a$} & \multicolumn{2}{|c|}{$c$} & \multicolumn{2}{|c|}{$u$} \\
\hline & \multicolumn{3}{|c|}{ Rutile } & \multirow[b]{2}{*}{$(-1.08)$} & \multirow[b]{2}{*}{0.304} & \multirow[b]{2}{*}{$(-0.33)$} \\
\hline PW-LDA SC (Ref. 14) & 4.555 & $(-0.70)$ & 2.922 & & & \\
\hline PW-LDA SC (Ref. 14) & 4.528 & $(-1.29)$ & 2.918 & $(-1.21)$ & 0.303 & $(-0.66)$ \\
\hline PW-LDA SC (Ref. 61) & 4.567 & $(-0.44)$ & 2.932 & $(-0.74)$ & 0.305 & $(0.00)$ \\
\hline PW-LDA LC (Ref. 60) & 4.536 & $(-1.11)$ & 2.915 & $(-1.32)$ & 0.304 & $(-0.33)$ \\
\hline PW-LDA LC (Ref. 59) & 4.653 & $(1.44)$ & 2.966 & $(0.41)$ & 0.305 & $(0.00)$ \\
\hline PW-LDA LC (Ref. 13) & 4.603 & $(0.35)$ & 2.976 & $(0.74)$ & 0.304 & $(-0.33)$ \\
\hline PW-LDA LC (Ref. 62) & 4.638 & $(1.11)$ & 2.923 & $(-1.05)$ & 0.305 & $(0.00)$ \\
\hline PW-GGA LC (Ref. 13) & 4.624 & $(0.81)$ & 2.992 & $(1.29)$ & 0.305 & $(0.00)$ \\
\hline PW-GGA LC (Ref. 64) & 4.690 & $(2.25)$ & 2.990 & $(1.22)$ & 0.306 & $(0.33)$ \\
\hline OLCAO-LDA AE (Ref. 66) & 4.622 & $(0.77)$ & 2.983 & $(0.99)$ & 0.304 & $(-0.33)$ \\
\hline LCAO-HF LC (Ref. 57) & 4.555 & $(-0.70)$ & 2.998 & $(1.49)$ & 0.306 & $(0.33)$ \\
\hline LCAO-HF AE (Ref. 58) & 4.560 & $(-0.59)$ & 3.022 & $(2.30)$ & 0.305 & $(0.00)$ \\
\hline LCAO-HF AE (Ref. 65) & 4.529 & $(-1.26)$ & 3.088 & ( 4.54$)$ & 0.305 & $(0.00)$ \\
\hline LCAO-HF AE (Ref. 65) & 4.548 & $(-0.85)$ & 2.993 & $(1.32)$ & 0.305 & $(0.00)$ \\
\hline LCAO-LDA AE (Ref. 63) & 4.529 & $(-1.26)$ & 2.942 & $(-0.41)$ & 0.304 & $(-0.33)$ \\
\hline X ray 298 K (Ref. 34) & 4.594 & $(0.15)$ & 2.958 & $(0.14)$ & 0.305 & $(0.00)$ \\
\hline Neutron 295 K (Ref. 37) & 4.593 & $(0.13)$ & 2.959 & $(0.17)$ & 0.305 & $(0.00)$ \\
\hline Neutron 15 K (Ref. 37) & 4.587 & & 2.954 & & 0.305 & \\
\hline \multicolumn{7}{|c|}{ Anatase } \\
\hline PW-LDA SC (Ref. 14) & 3.744 & $(-1.00)$ & 9.497 & $(-0.05)$ & 0.207 & $(-0.48)$ \\
\hline PW-LDA SC (Ref. 14) & 3.747 & $(-0.93)$ & 9.334 & $(-1.77)$ & 0.210 & $(-0.96)$ \\
\hline PW-LDA LC (Ref. 13) & 3.781 & $(-0.03)$ & 9.793 & ( 3.06$)$ & 0.204 & $(-1.92)$ \\
\hline LCAO-HF LC (Ref. 11) & 3.763 & $(-0.50)$ & 9.851 & $(3.67)$ & 0.202 & $(-2.88)$ \\
\hline X ray 301 K (Ref. 35) & 3.785 & $(0.08)$ & 9.514 & $(0.13)$ & 0.208 & $(0.00)$ \\
\hline Neutron 295 K (Ref. 37) & 3.785 & $(0.08)$ & 9.512 & $(0.11)$ & 0.208 & $(0.00)$ \\
\hline Neutron 15 K (Ref. 37) & 3.782 & & 9.502 & & 0.208 & \\
\hline
\end{tabular}

ions with a further two $\mathrm{O}$ ions slightly further away $[(6+2)$ fold coordination]. In light of these findings and the lack of reliable data for $\mathrm{TiO}_{2}$ at very high pressures, it seems possible that $\mathrm{TiO}_{2}$ may also adopt the $\mathrm{Pa} \overline{3}$ structure. To the authors' knowledge, this possibility has not been explored in previous work.

\section{G. Cotunnite}

The cotunnite $\left(\mathrm{PbCl}_{2}\right)$ structured $\mathrm{TiO}_{2}$ was recently discovered in high pressure diamond anvil experiments at pressures above $60 \mathrm{GPa}$. The unit cell of cotunnite is illustrated in Fig. 1. This structure is orthorhombic, belonging to the space group Pnma with the lattice vectors determined at 61 $\mathrm{GPa}$ to be $\mathbf{a}=5.163 \AA, \mathbf{b}=2.989 \AA$, and $\mathbf{c}=5.966 \AA$. There are four $\mathrm{TiO}_{2}$ formula units per unit cell with ions at $\pm\left(x, \frac{1}{4}, z ; x+\frac{1}{2}, \frac{1}{4}, \frac{1}{2}-z\right)$, where for the Ti ions, $x=0.264$ and $z=0.110$; for one $\mathrm{O}$ ion, $x=0.346$ and $z=0.422$; and for the second $\mathrm{O}$ ion, $x=0.012$ and $z=0.325$. The Ti ions are ninefold coordinated to $\mathrm{O}$ and the $\mathrm{O}$ ions form elongated tricapped trigonal prisms containing the titanium atoms.

\section{METHOD}

The calculations presented have been performed using the LCAO, all-electron, CRYSTAL98 software, ${ }^{40}$ with supplementary calculations performed using the PW pseudopotential software CASTEP. ${ }^{41}$

In the LCAO formalism implemented in CRYSTAL98, the crystalline orbitals are expanded as a linear combination of atom centered Gaussian orbitals with $s, p$, or $d$ symmetry (the basis set). All-electron (AE) calculations were performed in which there is no shape approximation to the potential or density. A variety of treatments of exchange and correlation were used: Hartree-Fock (HF) theory, where exchange is computed exactly but correlation is neglected, and density functional theory (DFT) using the local-density approximation $(\mathrm{LDA})^{42,43}$ and the generalized gradient approximation $(\mathrm{GGA}){ }^{44}$

The main numerical approximation in these calculations is the choice of the basis set. High quality all-electron basis sets developed and optimized for use in rutile $\mathrm{TiO}_{2}$ bulk and surface studies ${ }^{45,46}$ have been used. ${ }^{47} \mathrm{~A}$ basis set having three independent radial functions to describe each valence elec- 
tron is denoted as a triple-valence all-electron (TVAE) basis set. The TVAE basis set has been used extensively in previous studies of $\mathrm{TiO}_{2} \cdot{ }^{46,48-50}$ In the current study this basis set is supplemented by the addition of a $d$-symmetry polarization function on the $\mathrm{O}$ ions to produce the TVAE* basis set. While this additional flexibility has only a minor effect on the description of the rutile phase, it is found to be important for an accurate description of the anatase structure: the error in the c lattice constant of the latter is reduced from 5\% to $2 \%$. It has recently been demonstrated that although the TVAE* basis set describes structural properties consistently well, it is not sufficient to converge subtle differences in the energies of related structures. ${ }^{31,48}$ In order to converge the energy difference between phases we have thus used the TVAE** $^{*}$ basis set in which an additional diffuse $d$-symmetry function on the titanium ions is included ${ }^{47}$.

The sampling of $k$ space is also an important approximation. Pack-Monkhorst grids ${ }^{40,51}$ of shrinking parameters 4-8 were used depending on the phase being treated. Tests showed that this was sufficient to converge structures to better than $0.01 \AA$, total energies to $0.25 \mathrm{~kJ} / \mathrm{mol}$, and energy differences between phases to $0.025 \mathrm{~kJ} / \mathrm{mol}$.

A further approximation in the present study is related to the use of local basis functions. The truncation of the direct space summations of the Coulomb and exchange series is controlled by five Gaussian overlap criteria. The control of these approximations was described in detail elsewhere. ${ }^{40,52}$ Tests showed that using values of $10^{-7}, 10^{-6}, 10^{-7}, 10^{-7}$, and $10^{-14}$ results in a numerical error of around $2 \mathrm{~kJ} / \mathrm{mol}$ and less than $0.01 \AA$ per unit cell in the relative energies and structures of different phases. In the calculations comparing the delicate stability of rutile and anatase, we used very high tolerances (up to $10^{-9}, 10^{-8}, 10^{-9}, 10^{-9}$, and $10^{-16}$ ). The structural optimizations were converged to a displacement of less than $0.01 \AA$, or an energy difference of less than $10^{-4} \mathrm{~kJ} / \mathrm{mol}$ using a Broyden-Fletcher-Goldfarb-Shanno (BFGS) minimization algorithm. ${ }^{53}$

Supplementary PW-pseudopotential calculations were performed using the CASTEP software. ${ }^{41}$ The LDA and GGA functionals used were the same as for the LCAO calculations. The $\mathrm{Ti}(1 s, 2 s$, and $2 p)$ and $\mathrm{O}(1 s)$ core states were described using small-core (SC) ultrasoft pseudopotentials. ${ }^{54}$ Previous work has shown that large-core (LC) pseudopotentials that incorporate $\mathrm{Ti} 3 s$ and $3 p$ states can lead to significant errors in the calculation of structural and energetic properties. ${ }^{48}$ The sampling of $k$ space was performed using a Monkhorst-Pack net ${ }^{55}$ of shrinking factor 4. A range of plane-wave cutoff energies $\left(E_{c u t}\right)$ from 300 to $600 \mathrm{eV}$ was used to ensure convergence of the structure and relative energies of different phases to about $0.001 \AA$ and $2 \mathrm{~kJ} / \mathrm{mol}$ per unit cell, respectively. The calculations of $\mathrm{TiO}_{2}$ at high pressures were performed by applying an hydrostatic pressure and minimizing the enthalpy

$$
H=U+P V
$$

with respect to all structural parameters.

Bulk moduli have been computed by fitting to a BirchMurnaghan equation of state of the form ${ }^{56}$

$$
\begin{aligned}
P= & 1.5 K_{T}\left[\left(V_{0} / V\right)^{7 / 3}-\left(V_{0} / V\right)^{5 / 3} \times\left[1-0.75\left(4-K^{\prime}\right)\right.\right. \\
& \left.\times\left(V_{0} / V\right)^{2 / 3}-1\right] .
\end{aligned}
$$

For all phases, cell optimizations were performed for at least nine different pressures.

\section{RESULTS}

\section{A. Structure, phase stability, and elastic properties of rutile and anatase at $0 \mathrm{GPa}$}

Before discussing the high-pressure phases the relative stability of rutile and anatase at $0 \mathrm{GPa}$ is considered in some detail. Above $870 \mathrm{~K}$, anatase and brookite are found to transform irreversibly to rutile. ${ }^{6}$ The enthalpy change in the anatase to rutile transformation has been measured using calorimetric techniques. There are significant differences in the values reported in the literature: from the exothermic values of $\quad \Delta H_{298}=-11.7 \mathrm{~kJ} / \mathrm{mol},{ }^{7} \quad \Delta H_{968}=-6.56 \mathrm{~kJ} / \mathrm{mol}^{8}{ }^{8}$ $\Delta H_{971}=-3.26 \mathrm{~kJ} / \mathrm{mol},{ }^{6}$ and $\Delta H_{903}=-0.42 \mathrm{~kJ} / \mathrm{mol},{ }^{9}$ to the endothermic values of $\Delta H_{1183}=+11.66$ and $\Delta H_{298}$ $=+0.42 \mathrm{~kJ} / \mathrm{mol}^{10}$

As a prerequisite to determining the energetics of the two phases, it is important that fully unconstrained and carefully converged structures are obtained. In Table I, a summary of the results from a selection of experimental and recent $a b$ initio studies of the structural parameters of rutile and anatase is presented. Early HF, ${ }^{57,58} \mathrm{PW}-\mathrm{LDA},{ }^{59-62}$ and combined HF and DFT studies of rutile ${ }^{63}$ yielded lattice parameters to within $2 \%$ of experiment. More recent studies that have taken advantage of improvements in the theoretical techniques and available computing power to perform calculations with improved treatments of exchange and correlation (DFT calculations based on the GGA) (Refs. 13 and 64) and higher numerical accuracy (improved basis sets) (Ref. 65) have yielded results consistent with those from the earlier work. There have been only a few publications of the lattice parameters of anatase computed using ab initio methods. LCAO-HF (Ref. 11) and PW-LDA (Ref. 13) studies using large core pseudopotentials yielded structures with 3-4\% errors in the $\mathbf{c}$ lattice constant, but later work using small core pseudopotentials reduced these errors to $1-2 \%$. Studies of the energetics of rutile, anatase and brookite with the orthogonalized-linear-combinations-of-atomic-orbitals method within the LDA predicted reasonably accurate lattice parameters for rutile, but the other two phases were not relaxed fully. ${ }^{66}$

Generally speaking, all the $a b$ initio studies of rutile and anatase have yielded structural parameters to within a few percent of experiment. The structural parameters computed in the current study are given in Table II. While the present HF, LDA, and GGA calculations all yield lattice parameters in reasonable agreement with experiment, there are clear trends with regards to treatment of exchange and correlation. For rutile, HF theory tends to underestimate a and overestimate c, resulting in an overestimate of the cell volume, the LDA leads to an underestimate of both $\mathbf{a}$ and $\mathbf{c}$ and the GGA to an overestimate of both a and c. Similar trends are also seen for anatase. Although all the LCAO calculations over- 
TABLE II. The optimized structural parameters of rutile and anatase (in $\AA$ ) computed using ab initio methods. Numbers in parentheses indicate the percent deviation from experiment.

\begin{tabular}{|c|c|c|c|c|c|c|c|c|c|c|c|c|c|}
\hline \multirow[t]{2}{*}{ Method } & \multirow[t]{2}{*}{ Basis } & \multicolumn{6}{|c|}{ Rutile } & \multicolumn{6}{|c|}{ Anatase } \\
\hline & & \multicolumn{2}{|c|}{$a$} & \multicolumn{2}{|c|}{$c$} & \multicolumn{2}{|c|}{$u$} & \multicolumn{2}{|c|}{$a$} & \multicolumn{2}{|c|}{$c$} & \multicolumn{2}{|c|}{$u$} \\
\hline \multicolumn{14}{|l|}{ LCAO } \\
\hline \multirow[t]{2}{*}{$\mathrm{HF}$} & TVAE* ${ }^{*}$ & 4.575 & $(-0.26)$ & 2.999 & $(1.52)$ & 0.306 & $(0.33)$ & 3.781 & $(-0.03)$ & 9.735 & $(2.45)$ & 0.203 & $(-2.40)$ \\
\hline & TVAE** & 4.579 & $(-0.17)$ & 2.989 & ( 1.18$)$ & 0.306 & $(0.33)$ & 3.780 & $(-0.05)$ & 9.716 & $(2.25)$ & 0.203 & $(-2.40)$ \\
\hline \multirow[t]{2}{*}{ LDA } & TVAE* & 4.539 & $(-1.05)$ & 2.953 & $(-0.03)$ & 0.305 & $(0.00)$ & 3.739 & $(-1.14)$ & 9.694 & $(2.02)$ & 0.205 & $(-1.44)$ \\
\hline & TVAE** & 4.548 & $(-0.85)$ & 2.944 & $(-0.34)$ & 0.305 & $(0.00)$ & 3.729 & $(-1.40)$ & 9.695 & $(2.03)$ & 0.204 & $(-1.92)$ \\
\hline \multirow[t]{2}{*}{ GGA } & TVAE* & 4.627 & $(0.87)$ & 2.981 & $(0.91)$ & 0.305 & $(0.00)$ & 3.794 & $(0.32)$ & 9.712 & $(2.21)$ & 0.206 & $(-0.96)$ \\
\hline & TVAE** & 4.623 & ( 0.78$)$ & 2.987 & $(1.12)$ & 0.306 & $(0.33)$ & 3.801 & $(0.50)$ & 9.719 & ( 2.28$)$ & 0.206 & $(-0.96)$ \\
\hline \multicolumn{14}{|l|}{ PW } \\
\hline \multirow[t]{2}{*}{ LDA } & 340 & 4.574 & $(-0.28)$ & 2.927 & $(-0.91)$ & 0.304 & $(-0.33)$ & 3.758 & $(-0.63)$ & 9.495 & $(-0.07)$ & 0.208 & $(0.00)$ \\
\hline & 380 & 4.562 & $(-0.55)$ & 2.920 & $(-1.15)$ & 0.304 & $(-0.33)$ & 3.746 & $(-0.95)$ & 9.480 & $(-0.23)$ & 0.208 & $(0.00)$ \\
\hline \multirow[t]{2}{*}{ GGA } & 340 & 4.651 & $(1.40)$ & 2.964 & $(0.34)$ & 0.307 & $(0.66)$ & 3.792 & $(0.26)$ & 9.714 & $(2.23)$ & 0.206 & $(-0.96)$ \\
\hline & 380 & 4.641 & ( 1.18$)$ & 2.958 & $(0.14)$ & 0.305 & $(0.00)$ & 3.777 & $(-0.13)$ & 9.818 & ( 3.33$)$ & 0.205 & $(-1.44)$ \\
\hline
\end{tabular}

estimate c, the LCAO-LDA calculations yield the smallest overestimate. The structures in Table I computed using a LCAO formalism with an AE basis set show the same trends as the current results.

When making comparisons with the previous PW results, it is important to note that the pseudopotential approximation can have a significant effect on the computed structures. Calculations on bulk and surfaces of $\mathrm{TiO}_{2}$ polymorphs have demonstrated that the LC pseudopotential approximation tends to give results in significantly worse agreement with experiment compared to the SC pseudopotential. ${ }^{48}$ In the current study, all PW calculations have been performed using SC pseudopotentials, and give the same trends as the previous PW-SC and LCAO-AE results, whereas a number of PW calculations in Table I employed LC pseudopotentials and a large scatter is seen in the trends of these computed structures. For example, the PW-LC calculations of rutile performed with the LDA have predicted an underestimate of a and $\mathbf{c}^{60}$, an overestimate of $\mathbf{a}$ and $\mathbf{c},{ }^{13,59}$ and an overestimate of $\mathbf{a}$ but an underestimate of $\mathbf{c}^{62}$

For anatase, incorporating a $d$-symmetry polarization function on the $\mathrm{O}$ basis set leads to a significant improvement in the agreement with experiment. A thorough discussion of the influence of basis set on the computed structure of rutile and anatase was given elsewhere. ${ }^{31}$ As can be seen from Table II, a, c, and $\mathbf{u}$ are within $2 \%$ of experiment with $d$ functions, whereas the c parameter can be as much as $5 \%$ too large without it. This indicates that the $\mathrm{O}$ ion responds to its nonspherical environment with quadrupolar distortions which cannot be treated adequately using only $s$ - and $p$-symmetry functions.

An early comparison of the energetics of rutile and anatase using LCAO-HF theory with smaller basis sets than that used in the current study yielded structural parameters in good agreement with experiment and found anatase to be the lower-energy phase by $2.51 \mathrm{~kJ} / \mathrm{mol}^{11}$ The addition of a posteriori correlation based on DFT (Ref. 12) reversed this trend, yielding rutile as the more stable phase by between 2.96 and $11.50 \mathrm{~kJ} / \mathrm{mol}$, depending on the correlation functional used.
PW-LDA and PW-GGA calculations using LC pseudopotentials ${ }^{13}$ predicted the structure of the rutile, anatase, brookite, and columbite phases to within $2 \%$ of experiment. However, these calculations were in disagreement with regard to the phase stability of rutile and anatase. The LDA calculations predict rutile to be more stable than anatase by $2.11 \mathrm{~kJ} / \mathrm{mol}$, whereas the GGA calculations yield anatase as the more stable phase by $4.82 \mathrm{~kJ} / \mathrm{mol}$.

A recent PW-LDA study found that the relative stability of the two phases is sensitive to the pseudopotential even when SC pseudopotentials are used. ${ }^{14}$ Two different SC pseudopotentials were tested, with one type yielding anatase as the more stable form by $5.852 \mathrm{~kJ} / \mathrm{mol}$ (Troulier Martin pseudopotential) and the other predicting rutile to be the more stable form by $4.598 \mathrm{~kJ} / \mathrm{mol}$ (Teter pseudopotential). It is clear that the predicted relative stability of the two phases is very sensitive to the numerical approximations and to the treatment of exchange and correlation adopted.

In Table III the influence of the basis set on the computed energy difference between rutile and anatase is reported. As the basis set is improved, the total energy varies significantly but the energy differences are far less sensitive with the anatase phase clearly the more stable. The PW calculations performed in the current study with a SC pseudopotential also

TABLE III. Calculated energy difference ( $\delta E=E_{\text {rutile }}$ $\left.-E_{\text {anatase }}\right)$ between rutile and anatase.

\begin{tabular}{lccc}
\hline \hline Method & \multicolumn{3}{c}{$\delta E(\mathrm{~kJ} / \mathrm{mol})$} \\
\hline LCAO & \multicolumn{3}{c}{ Basis set } \\
& TVAE* $^{*}$ & TVAE** $^{* *}$ \\
HF & 2.72 & & 4.25 \\
LDA & -0.32 & & 1.88 \\
GGA & 5.46 & & 7.59 \\
PW & & $\mathrm{E}_{\text {cut }}(\mathrm{eV})$ & \\
& 340 & & 380 \\
LDA & 3.31 & & 2.13 \\
GGA & 7.17 & & 9.68 \\
\hline \hline
\end{tabular}


TABLE IV. The bulk moduli (in GPa) of the various phases of $\mathrm{TiO}_{2}$ computed in the current work with the TVAE* basis set using HF and LDA treatments of exchange and correlation. The LCAO-HF (Refs. 63 and 65) and LCAO-LDA (Ref. 63) calculations have been performed using the CRYSTAL98 package.

\begin{tabular}{|c|c|c|c|c|c|c|c|}
\hline Method & Rutile & Anatase & Columbite & Baddeleyite & Pyrite & Fluorite & Cotunnite \\
\hline LCAO-HF & $239 \pm 10$ & $202 \pm 10$ & $264 \pm 10$ & $300 \pm 10$ & $318 \pm 10$ & $331 \pm 10$ & $380 \pm 10$ \\
\hline LCAO-LDA & $241 \pm 10$ & $195 \pm 10$ & & & $273 \pm 10$ & $308 \pm 10$ & \\
\hline LCAO-HF (Ref. 63) & 281 & & & & & & \\
\hline LCAO-LDA (Ref. 63) & 264 & & & & & & \\
\hline LCAO-HF (Ref. 65) & 304 & & & & & & \\
\hline LCAO-HF (Ref. 65) & 236 & & & & & & \\
\hline LCAO-LDA (Ref. 66) & 209 & 272 & & & & & \\
\hline PW-LDA (Ref. 59) & 240 & & & & & & \\
\hline PW-LDA (Ref. 30) & 243 & 194 & 247 & 249 & & 282 & \\
\hline PW-LDA (Ref. 13) & 244 & 190 & 215 & & & 287 & \\
\hline $\mathrm{X}$ ray (Ref. 33) & & $178 \pm 1$ & & & & & \\
\hline$X$ ray (Ref. 25) & & $179 \pm 2$ & $258 \pm 8$ & $290 \pm 10$ & & & \\
\hline$X$ ray (Ref. 85) & & & $253 \pm 4$ & & & & \\
\hline $\mathrm{X}$ ray (Ref. 21) & & 59 & 98 & 522 & & & \\
\hline $\mathrm{X}$ ray (Ref. 78) & $211 \pm 7$ & & & & & & \\
\hline$X$ ray (Ref. 22) & & 360 & & & & & \\
\hline$X$ ray (Ref. 23) & $230 \pm 20$ & & $260 \pm 30$ & $290 \pm 20$ & & & \\
\hline $\mathrm{X}$ ray (Ref. 5) & & $178 \pm 1$ & & $304 \pm 6$ & & & $431 \pm 10$ \\
\hline
\end{tabular}

predict anatase to be the more stable form with the LDA and GGA relative stabilities in excellent agreement with the results from the LCAO calculations, performed using the

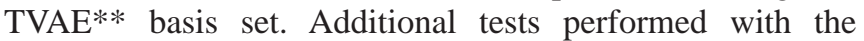
CASTEP code using very high-energy cutoffs (up to $600 \mathrm{eV}$ ) did not have a significant influence on either the order of the phases or the magnitude of the stability.

As noted above, a previous HF study found anatase to be the more stable phase but addition of a posteriori correlation corrections reversed this trend. ${ }^{11}$ We have calculated the "correlation corrected" total energies of rutile and anatase using the $\mathrm{TVAE}^{* *}$ basis set within the HF approximation using all the available exchange-correlation functionals in CRYSTAL98. Using a variety of LDA (Perdew-Zunger, ${ }^{43}$ Vosko-Wilk-Nusair, ${ }^{67}$ Von Barth-Hedin, ${ }^{68}$ and Perdew-Wang ${ }^{69-71}$ ) and GGA (Colle-Salvetti, ${ }^{72}$ Wigner-Levy, ${ }^{73}$ Perdew $86,{ }^{74}$ Perdew $91,{ }^{75}$ Perdew-Wang, ${ }^{69-71,75}$ Becke, ${ }^{76}$ Perdew-Burke-Ernzerhof, ${ }^{44}$ and Lee-Yang-Parr ${ }^{77}$ ) functionals as a posteriori corrections to the HF total energy yields a value of $\delta E_{\text {rutile-anatase }}$ of between 3.1 and $3.5 \mathrm{~kJ} / \mathrm{mol}$ for LDA functionals and -1.5 and $-9.5 \mathrm{~kJ} / \mathrm{mol}$ for GGA functionals. Clearly, the use of selfconsistent exchange and correlation is required for reliable results.

The computed bulk moduli of rutile and anatase and the phases discussed in Sec. IV B are presented in Table IV. The bulk modulus of rutile has been measured to be about 210 $\mathrm{GPa},{ }^{78-80}$ with more recent studies verifying this value. ${ }^{23,81}$ Until recently, there was considerable disagreement in the literature as to even the approximate magnitude of the experimental bulk modulus of anatase, with measurements ranging from $59 \mathrm{GPa}$ (Ref. 21) to $360 \mathrm{GPa}^{22}$ Two recent independent measurements have clarified the matter: both experiments yielded a bulk modulus of around $180 \mathrm{GPa}^{25,82}$ Arlt et $a .^{25}$ pointed out that the use of single crystal as opposed to powder samples can have an influence on the measured bulk modulus but this effect was found to be within the experimental error bars (179 GPa for a singlecrystal sample and $190 \pm 10 \mathrm{GPa}$ for a powder sample). The computed bulk modulus of rutile in the current work is in good agreement with experiment being only around $10 \%$ too high and is in excellent agreement with previous PW studies. ${ }^{13,30,59}$ The discrepancy between the rutile bulk modulus computed in this study and that from the recent allelectron LCAO studies using very similar computational techniques is due to the fitting procedure used to compute the bulk modulus. In the current study, the Birch-Murnaghan equation of state ${ }^{56}$ has been used to extract the bulk modulus, whereas the values from the previous LCAO studies were computed from a polynomial fit of the energy-volume curve. Computing the bulk modulus with the fitting procedure described in Ref. 63, values of $277 \mathrm{GPa}(\mathrm{HF})$ and $253 \mathrm{GPa}$ (LDA) were obtained. Clearly, the fitting procedure has a significant influence on the predicted modulus. The BirchMurnaghan equation of state is used in this study because most experimental studies use this equation of state to extract bulk moduli from pressure-volume data.

The bulk modulus of anatase is calculated to be lower than that of rutile (by about $15 \%$ ), in agreement with experiment. The computed bulk modulus of about $200 \mathrm{GPa}$ is in reasonable agreement with previous PW-LDA calculations which found it to be about $190 \mathrm{GPa}$ (Refs. 13 and 30) and the experimental value of $180 \mathrm{GPa}^{25,82}$

The calculations of Mo and Ching, ${ }^{66}$ which predicted anatase to have a higher bulk modulus than rutile, were performed under the constraint of a fixed c/a ratio during opti- 


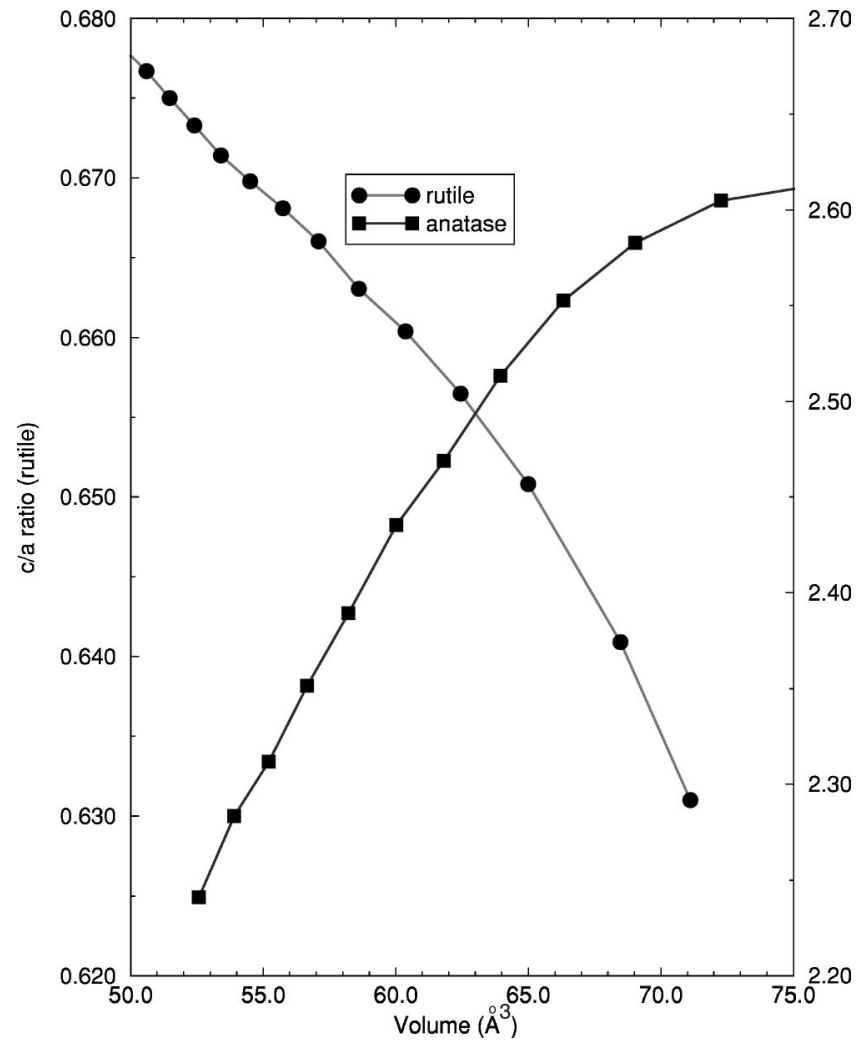

FIG. 2. The change in $c / a$ ratio with cell volume of rutile and anatase computed within the HF approximation.

mization of the volume at a given applied pressure. However, the current calculations demonstrate that rutile and anatase both undergo anisotropic compression. The computed change in the c/a ratio with pressure for the two phases is illustrated in Fig. 2. The ratio increases for rutile and decreases for anatase as the cell volume is reduced. The longer cell vector (a for rutile and $\mathbf{c}$ for anatase) is the more compressible in both structures, in agreement with experimental observations ${ }^{21,23,33,78,83}$ (see Fig. 3). Constraining the unit cell to a fixed c/a ratio leads to significant errors in the computed structure and bulk modulus.

\section{B. High-pressure phases}

$\mathrm{TiO}_{2}$ has a rich phase diagram and forms a number of high-pressure phases, as described in Sec. I. Many of these phases have not been well characterized. For example, the existence of the fluorite phase of $\mathrm{TiO}_{2}$ has only been tentatively assigned, and it is not certain whether the fluorite and pyrite phases can exist between baddeleyite and cotunnite or if they are post-cotunnite. Although there have been theoretical calculations of the fluorite structure ${ }^{13,30}$ there have been no predictions of the structure or energetics of the pyrite or cotunnite phases. In order to understand the high-pressure phase diagram of $\mathrm{TiO}_{2}$, we have performed HF calculations of the rutile, anatase, columbite, baddeleyite, fluorite, pyrite, and cotunnite phases at pressures up to at least $70 \mathrm{GPa}$.

The predicted structures for the post-rutile and postanatase phases are presented in Table V. The calculated cell parameters for columbite at $0 \mathrm{GPa}$ are in excellent agreement
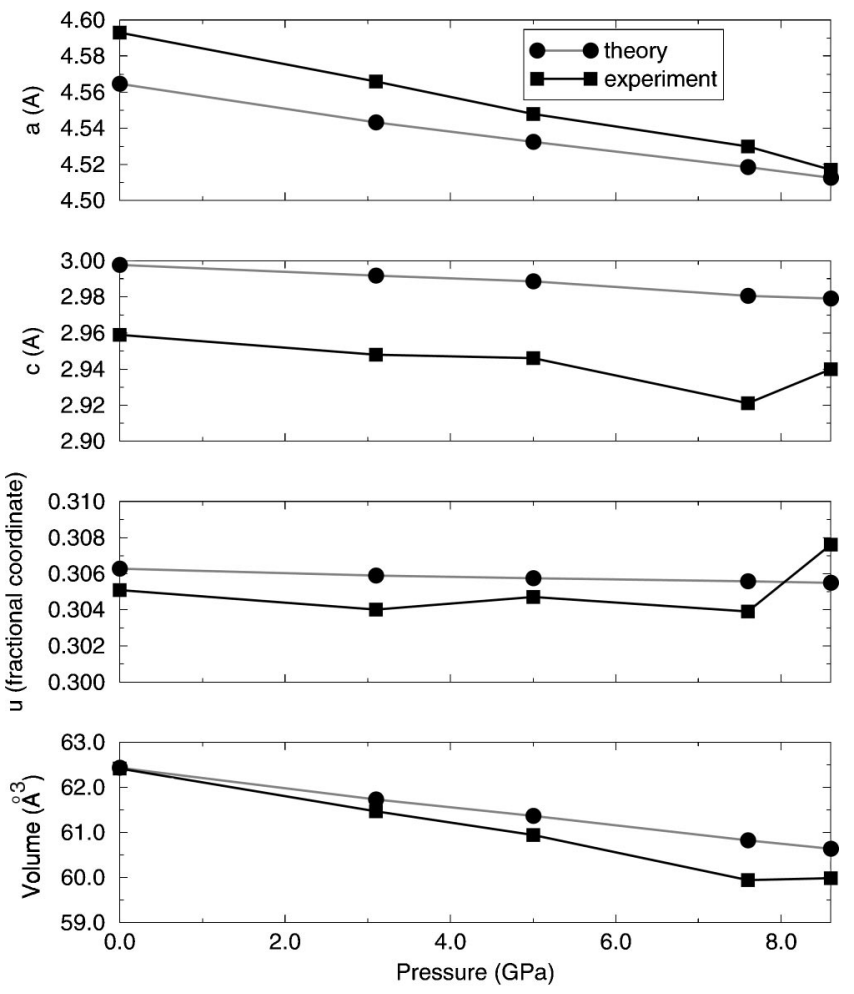

FIG. 3. Effect of pressure on the lattice parameters of rutile $\mathrm{TiO}_{2}$ computed within the HF approximation. Experimental data are from Ref. 83.

with previous results from LDA calculations and x-raydiffraction experiments. The $\mathbf{a}, \mathbf{b}$, and $\mathbf{c}$ lattice parameters computed with HF and LDA methods are within $1 \%$ of experiment. The predicted cell volume is overestimated by the HF methods (by $0.34 \%$ ) and underestimated by the LDA (by $0.32 \%$ ); these trends are consistent with those calculated for the rutile and anatase phases (Sec. IV A). The current HF and LDA calculations predict internal coordinates in excellent agreement with experimental observations $\mathrm{s}^{23}$ and in reasonable agreement with the results of recent PW-LDA calculations. $^{30}$

For baddeleyite, the computed lattice vectors are in excellent agreement (within around $1 \%$ ) with those observed. ${ }^{26}$ To our knowledge, there have been no experimental determination of the internal coordinates of this phase. The lattice vectors and internal coordinates of cotunnite have recently been measured, ${ }^{5}$ and the current calculations are in agreement with this data (see Table V). For the pyrite and fluorite phases, no experimental data on their structures are available; the structure computed here for the fluorite phase is in reasonable agreement with previous ab initio studies. ${ }^{13,30}$

The computed bulk moduli, along with the known experimental determinations for all the phases considered in this paper, are presented in Table IV. The bulk modulus of columbite $\mathrm{TiO}_{2}$ was measured in an early study to be 98 $\mathrm{GPa},{ }^{21}$ but more recent work has reported it to be somewhat higher than that of rutile; $250-260 \mathrm{GPa}^{23,85}$ The current HF calculations predict a value of $264 \mathrm{GPa}$, in good agreement with the most recent PW-LDA calculations $(247 \mathrm{GPa}){ }^{13}$ but somewhat higher than an earlier study which found it to be 
TABLE V. The structural parameters of the high-pressure phases of $\mathrm{TiO}_{2}$ (lengths in $\AA$ and angles in degrees) computed at $0 \mathrm{GPa}$ (unless specified) with the TVAE* basis set. The cell volume is in $\AA^{3}$ for two $\mathrm{TiO}_{2}$ formula units.

\begin{tabular}{|c|c|c|c|c|c|c|c|c|c|}
\hline & \multirow[t]{2}{*}{$a$} & \multirow[t]{2}{*}{$b$} & \multirow[t]{2}{*}{$c$} & \multirow[t]{2}{*}{$\beta$} & \multicolumn{4}{|c|}{ Internal coordinates } & \multirow[t]{2}{*}{ Volume } \\
\hline & & & & & & $x$ & $y$ & $z$ & \\
\hline & \multicolumn{9}{|c|}{ Columbite } \\
\hline \multirow[t]{2}{*}{$\mathrm{HF}$} & 4.553 & 5.468 & 4.932 & 90.0 & $\mathrm{Ti}$ & 0.000 & 0.169 & 0.250 & 61.393 \\
\hline & & & & & $\mathrm{O}$ & 0.274 & 0.385 & 0.420 & \\
\hline \multirow[t]{2}{*}{ LDA } & 4.528 & 5.438 & 4.954 & 90.0 & $\mathrm{Ti}$ & 0.000 & 0.170 & 0.250 & 60.993 \\
\hline & & & & & $\mathrm{O}$ & 0.274 & 0.381 & 0.418 & \\
\hline \multirow[t]{2}{*}{ LDA (Ref. 30) } & & Not Given & & 90.0 & $\mathrm{Ti}$ & 0.000 & 0.178 & 0.250 & 61.887 \\
\hline & & & & & $\mathrm{O}$ & 0.259 & 0.429 & 0.439 & \\
\hline \multirow[t]{2}{*}{ LDA (Ref. 13) } & 4.569 & 5.481 & 4.929 & 90.0 & $\mathrm{Ti}$ & 0.000 & 0.174 & 0.250 & 61.718 \\
\hline & & & & & $\mathrm{O}$ & & Not Given & & \\
\hline \multirow[t]{3}{*}{ Expt (Ref. 23) } & 4.541 & 5.493 & 4.906 & 90.0 & $\mathrm{Ti}$ & 0.000 & 0.171 & 0.250 & 61.187 \\
\hline & & & & & $\mathrm{O}$ & 0.286 & 0.376 & 0.412 & \\
\hline & \multicolumn{9}{|c|}{ Baddeleyite } \\
\hline \multirow{3}{*}{$\begin{array}{l}\mathrm{HF} \\
(20 \mathrm{GPa})\end{array}$} & 4.691 & 4.820 & 4.851 & 98.38 & $\mathrm{Ti}$ & 0.275 & 0.040 & 0.206 & 54.251 \\
\hline & & & & & $\mathrm{O}(1)$ & 0.079 & 0.350 & 0.328 & \\
\hline & & & & & $\mathrm{O}(2)$ & 0.444 & 0.760 & 0.482 & \\
\hline \multirow{3}{*}{$\begin{array}{l}\text { LDA (Ref. 13) } \\
(0 \mathrm{GPa})\end{array}$} & 4.790 & 4.915 & 4.924 & 99.97 & $\mathrm{Ti}$ & 0.279 & 0.047 & 0.209 & 57.092 \\
\hline & & & & & $\mathrm{O}(1)$ & 0.078 & 0.354 & 0.329 & \\
\hline & & & & & $\mathrm{O}(2)$ & 0.444 & 0.761 & 0.485 & \\
\hline \multirow{4}{*}{$\begin{array}{l}\text { Expt (Ref. 26) } \\
(20.3 \mathrm{GPa})\end{array}$} & 4.64 & 4.76 & 4.81 & 99.2 & $\mathrm{Ti}$ & & & & 52.435 \\
\hline & & & & & $\mathrm{O}(1)$ & & Not Given & & \\
\hline & & & & & $\mathrm{O}(1)$ & & & & \\
\hline & & & & & Cotunn & & & & \\
\hline \multirow{3}{*}{$\begin{array}{l}\mathrm{HF} \\
(60 \mathrm{GPa})\end{array}$} & 5.046 & 2.966 & 5.884 & 90.0 & $\mathrm{Ti}$ & 0.246 & 0.25 & 0.114 & 44.031 \\
\hline & & & & & $\mathrm{O}(1)$ & 0.358 & 0.25 & 0.427 & \\
\hline & & & & & $\mathrm{O}(2)$ & 0.026 & 0.75 & 0.337 & \\
\hline \multirow{4}{*}{$\begin{array}{l}\text { Expt (Ref. 5) } \\
\text { (61 GPa) }\end{array}$} & 5.163 & 2.989 & 5.966 & 90.0 & $\mathrm{Ti}$ & 0.264 & 0.25 & 0.110 & 46.034 \\
\hline & & & & & $\mathrm{O}(1)$ & 0.346 & 0.25 & 0.422 & \\
\hline & & & & & $\mathrm{O}(2)$ & 0.012 & 0.75 & 0.325 & \\
\hline & \multicolumn{9}{|c|}{ Fluorite } \\
\hline $\mathrm{HF}$ & 4.794 & 4.794 & 4.794 & 90.0 & $\mathrm{O}$ & 0.25 & 0.25 & 0.25 & 55.089 \\
\hline LDA & 4.748 & 4.748 & 4.748 & 90.0 & $\mathrm{O}$ & 0.25 & 0.25 & 0.25 & 54.745 \\
\hline GGA & 4.897 & 4.897 & 4.897 & 90.0 & $\mathrm{O}$ & 0.25 & 0.25 & 0.25 & 58.706 \\
\hline LDA (Ref. 30) & 4.860 & 4.860 & 4.860 & 90.0 & $\mathrm{O}$ & 0.25 & 0.25 & 0.25 & 57.397 \\
\hline \multirow[t]{2}{*}{ LDA (Ref. 13) } & 4.800 & 4.800 & 4.800 & 90.0 & $\mathrm{O}$ & 0.25 & 0.25 & 0.25 & 55.296 \\
\hline & & & & & Pyrit & & & & \\
\hline $\mathrm{HF}$ & 4.860 & 4.860 & 4.860 & 90.0 & $\mathrm{O}$ & 0.338 & 0.338 & 0.338 & 57.396 \\
\hline LDA & 4.801 & 4.801 & 4.801 & 90.0 & $\mathrm{O}$ & 0.340 & 0.340 & 0.340 & 55.314 \\
\hline GGA & 4.894 & 4.894 & 4.894 & 90.0 & $\mathrm{O}$ & 0.338 & 0.338 & 0.338 & 58.592 \\
\hline
\end{tabular}

$215 .^{30}$ The bulk modulus of the baddeleyite phase has been determined several times, with the most recent studies giving a value around $300 \mathrm{GPa}^{5,25,23}$ Haines and Lèger ${ }^{21}$ reported a value of $522 \mathrm{GPa}$, although there is serious doubt about the accuracy of this datum as only a few points were used to fit the Birch-Murnaghan equation of state; this study also reported very low bulk moduli for the anatase and columbite phases (59 and $98 \mathrm{GPa}$ ), in stark contrast to other studies.

For the pyrite, fluorite, and cotunnite phases, the computed HF bulk moduli are 318, 331, and $380 \mathrm{GPa}$. There have been no previous determinations of the bulk modulus of the pyrite phase, but the fluorite phase has been modeled with its bulk modulus predicted to be around 280-290 GPa. ${ }^{13,30}$ Calculations of the bulk modulus of the fluorite phase using the LDA yield a lower value of $308 \mathrm{GPa}$, in reasonably good agreement with previous PW-LDA studies. As can be seen from Table IV, HF theory tends to yield a bulk modulus higher than the LDA value, consistent with the results of a recent comparative study of the effect of the treatment of exchange and correlation on the computed bulk modulus. ${ }^{86}$ For the cotunnite phase, the computed bulk modulus is about $10 \%$ lower than that observed. 


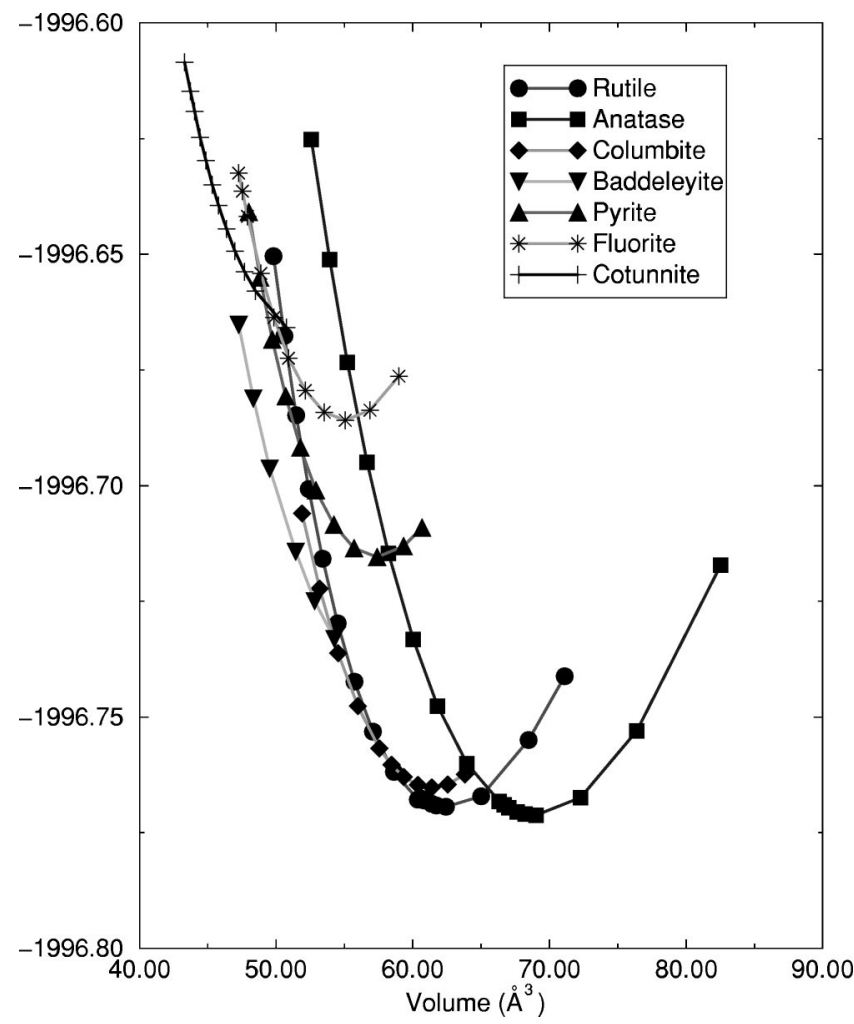

FIG. 4. The internal energy (in atomic units for two $\mathrm{TiO}_{2}$ formula units) with respect to volume for the $\mathrm{TiO}_{2}$ polymorphs calculated within the HF approximation using the TVAE* basis set.

There is an interesting trend for the HF approximation to overestimate the bulk modulus of low-pressure phases and to underestimate it in very high-pressure phases. In the case of rutile and anatase, HF theory tends to overestimate the bulk modulus by about $10 \%$; it is overestimated by less than $5 \%$ for columbite; for baddeleyite, it is within the error bars of the experimental value; and is about $10 \%$ lower than the experimental value for the cotunnite phase. One expects HF theory to overestimate bulk moduli in wide-band-gap insulators as the effect of electron correlation in these systems is to reduce effective ionic radii and to generate a weak additional binding term. Computed lattice constants thus tend to be somewhat larger than those observed and bulk moduli rather high. This is the opposite behavior to that expected in smallband-gap semiconductors, where correlation effects are dominated by the mixing of excited-state determinants with the HF ground state. It may be that the trend in the predicted bulk modulus in $\mathrm{TiO}_{2}$ with pressure is related to the closure of the band gap in the higher pressure phases.

The computed equations of state of the phases studied here are illustrated in Figs. 4 and 5. The present calculations predict that anatase to columbite transformation occurs at about $3.5 \mathrm{GPa}$, while the rutile to columbite transformation occurs at about $21 \mathrm{GPa}$. This result is in reasonable agreement with experimental observations, correctly predicting that anatase will undergo a phase transformation at lower pressures than rutile. Columbite is the most stable phase up to $31 \mathrm{GPa}$ where the calculations predict a transformation to baddeleyite which in turn transforms to cotunnite at $63 \mathrm{GPa}$.

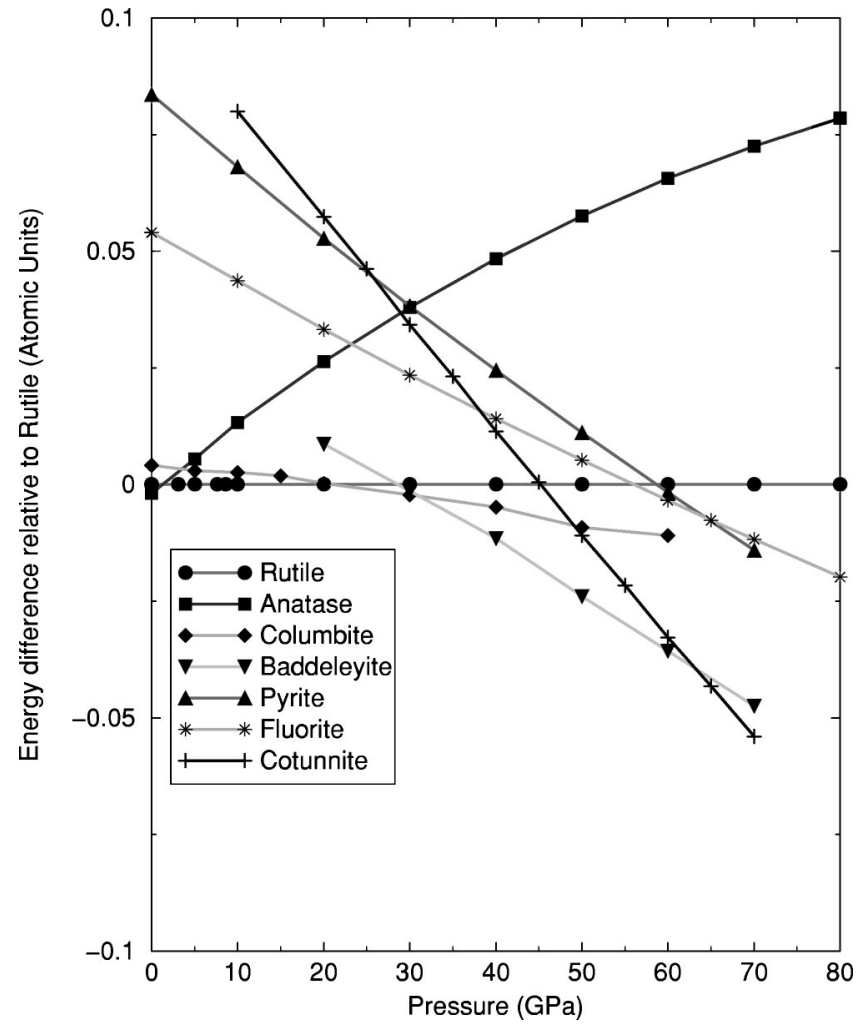

FIG. 5. The enthalpy difference, relative to rutile, for two $\mathrm{TiO}_{2}$ formula units with respect to pressure for the $\mathrm{TiO}_{2}$ polymorphs.

At no point between 0 and $70 \mathrm{GPa}$ is either the pyrite or fluorite phase predicted to be the most stable form, although the pyrite form is more stable than the fluorite structure up to $65 \mathrm{GPa}$. Extrapolation of the enthalpy versus pressure plots in Fig. 5 to higher pressures indicates that it is unlikely that pyrite or fluorite will become more stable than cotunnite at pressures below $100 \mathrm{GPa}$.

\section{DISCUSSION}

A number of general conclusions can be drawn from the large number of detailed calculations of the structure and energetics of $\mathrm{TiO}_{2}$ polymorphs presented above. First, the $\mathrm{HF}$ and GGA treatments of exchange and correlation tend to overestimate the cell volume, whereas the LDA tends to underestimate it. For HF theory this is related to the neglect of electron correlation, which results in an overestimate of the cell volume for wide-band-gap insulators, as discussed above. The LDA has a tendency to overbind molecules and solids, and in general underestimates cell volumes in these systems. The GGA overcorrects for this effect and, in these systems, yields cell volumes similar to those computed in the HF approximation.

The form of the basis set (PW or LCAO) has little influence on these structural trends as long as the total energy is reasonably well converged with respect to the basis set, and an all-electron approach or small-core pseudopotentials are used. Improving the LCAO basis sets from TVAE* to TVAE $^{* *}$ or increasing the plane-wave cutoff from 340 to $600 \mathrm{eV}$ has little effect on the predicted structures. However, 


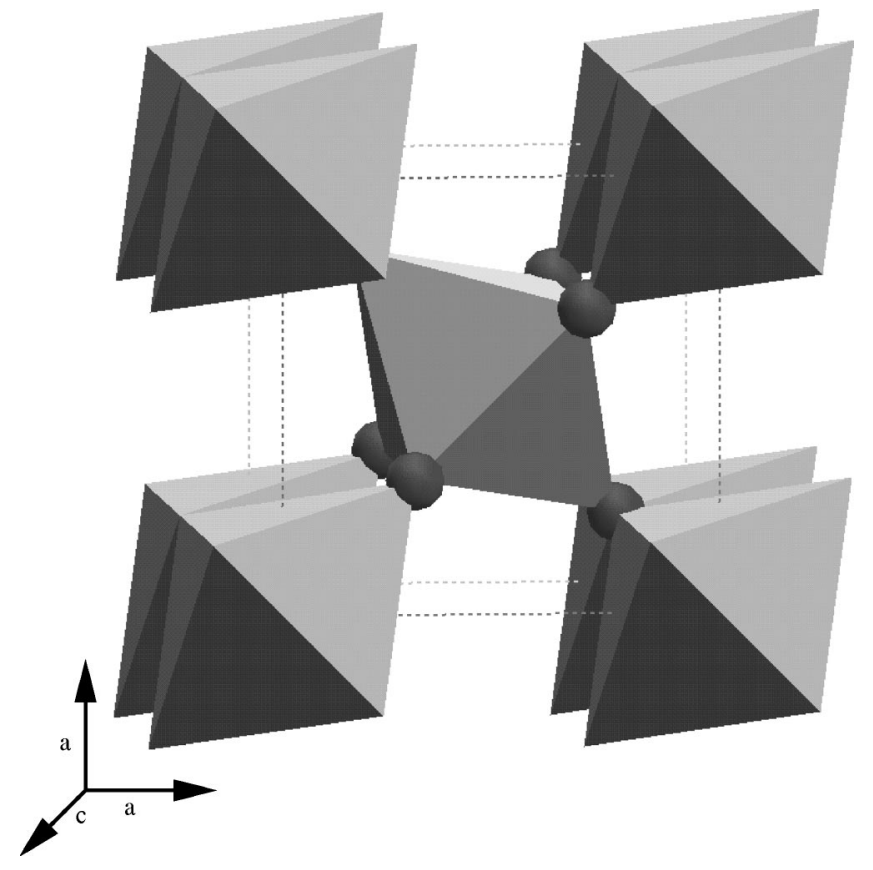

FIG. 6. The packing of $\mathrm{TiO}_{6}$ octahedra in rutile.

the calculation of the relative phase stability is sensitive to numerical and theoretical approximations, and great care is required when comparing two phases with very similar energies such as rutile and anatase at $0 \mathrm{GPa}$. The energy differences are only converged with the largest basis sets used here. Clearly, errors due to the numerical approximations may influence the predicted pressures of phase transformations. This effect can be estimated. The data in Table III indicate that the improvement of the basis set can lead to a difference of the relative phase stability of up to $2 \mathrm{~kJ} / \mathrm{mol}$. An error of such magnitude has a small effect on the computed transformation pressure where the gradient of the pressure versus enthalpy data of two phases differs significantly, as in the case of columbite and baddeleyite where this leads to an error of around $\pm 2 \mathrm{GPa}$ (see Fig. 5). However, for phases with very similar gradients, such as rutile and columbite, the error can be as large as $\pm 6 \mathrm{GPa}$. Thus the pressures of transformation predicted here may be somewhat different to those observed, but the sequence of the phase transformations is unlikely to be affected by these numerical errors.

The variation in elastic properties and the anisotropic compression of the different phases can be understood by considering the atomic structure of these phases and in particular the packing of the $\mathrm{TiO}_{6}$ octahedra. These octahedra are fairly rigid units which appear to behave in a rather consistent way in the rutile, anatase and columbite phases (see Fig. 9). However, their packing differs considerably.

In the rutile unit cell, the $\mathrm{TiO}_{6}$ octahedra pack in a regular arrangement with the neighboring octahedra sharing corners in the ab planes and edges in the c direction as depicted in Fig. 6. The corner-sharing octahedra have one Ti-O bond linking them, whereas edge-sharing octahedra share two Ti-O bonds. The crystal is thus more compressible in the ab plane where external stress can be taken up by the hinging of the octahedra than in the c direction which is supported by
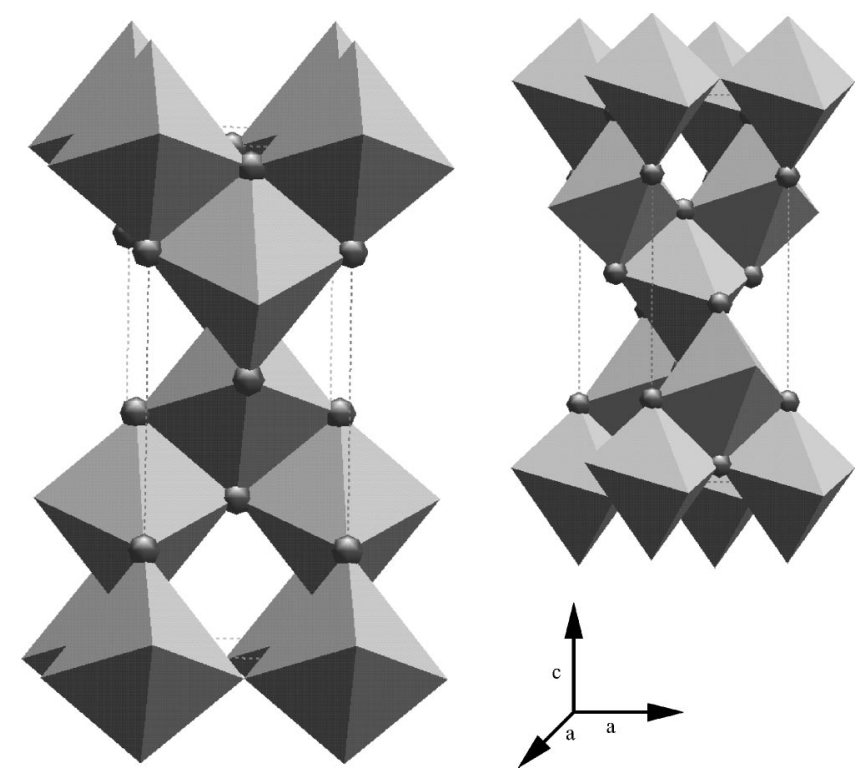

FIG. 7. The packing of $\mathrm{TiO}_{6}$ octahedra in anatase. The smaller image is a rotated view showing the zigzag arrangement of the octahedra along the a direction.

relatively inflexible "pillars" of edge-sharing octahedra.

In anatase, there are crossed rows of zigzagging octahedra that run along the $\mathbf{a}$ and $\mathbf{b}$ lattice vectors (see Fig. 7). The octahedra share four edges but the edges that are shared do not lie on opposite ends of the octahedron as they do in rutile. This has implications for the compressibility of the structure because compression can be accommodated by the octahedra hinging over each other more easily than in the rutile structure. This results in the computed bulk modulus of anatase being significantly lower than that of rutile.

Using similar simple models of the structure of these phases, the pressure-induced phase transformations of anatase and rutile to columbite can be understood by considering the nature of the electrostatic interactions of the ions in each structure. The energetics of the crystal are finely balanced between bringing as many oppositely charged ions as close together as possible while keeping like-charged ions as far apart as possible. Hence the lowest-energy structure is that which minimizes the cation-cation (Ti-Ti) and anion-anion $(\mathrm{O}-\mathrm{O})$ repulsions while maximizing the cation-anion attraction (Ti-O).

As noted in Sec. II, the rutile, anatase, and columbite structures are all built up from different arrangements of $\mathrm{TiO}_{6}$ octahedra. The biggest difference between the octahedral packing in rutile and in columbite, illustrated in Fig. 8, is that the octahedra in rutile form linear chains along the $\mathbf{c}$ direction whereas in columbite, they form zigzagging chains. $^{38}$ In the case of rutile and anatase, the nearest Ti-Ti and $\mathrm{O}-\mathrm{O}$ bond distances are shorter than in columbite, but the average octahedral Ti-O bond distances and octahedral volumes are similar (see Fig. 9). Compression of rutile and anatase leads to a reduction in the Ti-Ti and O-O bond distances. This reduction in bond distances can be compensated for by rearranging the octahedra and transforming to a columbite structure. 

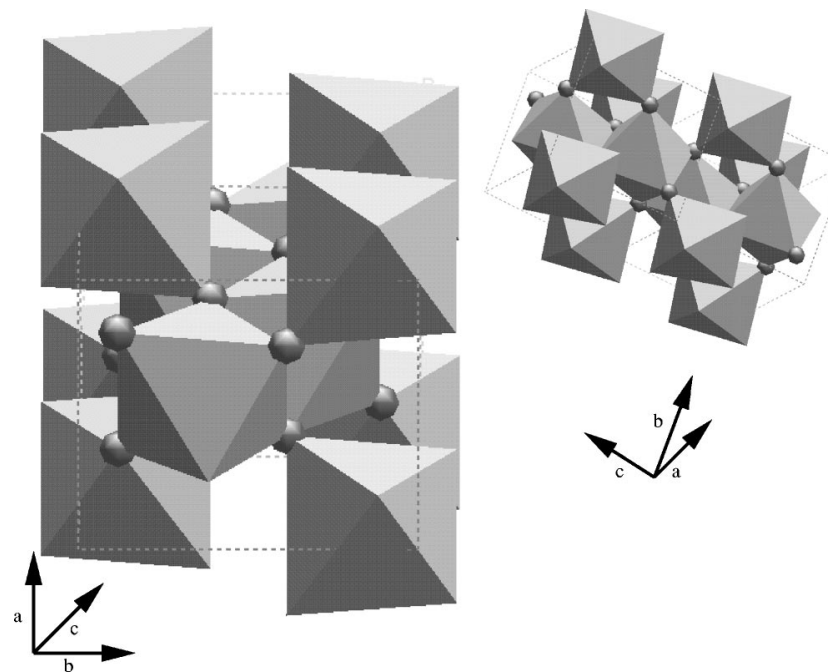

FIG. 8. The packing of $\mathrm{TiO}_{6}$ octahedra in columbite. The smaller image is a rotated view illustrating the packing of octahedra along the $\mathbf{c}$ direction.

Furthermore, the internal coordinate of the $\mathrm{Ti}$ ion in columbite can move along the $y$ Cartesian direction (corresponding to moving along the $\mathbf{b}$ lattice vector) but in rutile, it is fixed by symmetry. This degree of freedom results in a significantly larger Ti-Ti distance (see Fig. 10) than would occur if the material was in the rutile phase or if this coordinate was fixed. This feature of the packing also explains the anisotropic compressibility of columbite $\mathrm{TiO}_{2}$. The change in the unit cell parameters for a range of pressures is illustrated in Fig. 11. By far the most compressible direction is along the $\mathbf{b}$ lattice vector due to the freedom of the Ti ions to move along this direction. The $\mathbf{c}$ direction is also rather compressible because the octahedra can slide over each other somewhat due to the movement of the cations along the $\mathbf{b}$ direction. Finally, the a direction is the least compressible despite having only corner-sharing octahedra along it. The freedom of movement of the octahedra along the $\mathbf{b}$ and $\mathbf{c}$ directions is not evident along $\mathbf{a}$, and hence this direction is the stiffest. The order of compressibility along each lattice vector is in excellent agreement with experimental observations. $^{21}$

Finally, the post-columbite phase transformations can also be rationalized using simple models concerning the effect of pressure on the ionic size. The phase transformation from columbite to baddeleyite and cotunnite structures is probably due to changes in the radius ratio of the $\mathrm{Ti}$ and $\mathrm{O}$ ions. Oxygen ions are significantly more compressible than $\mathrm{Ti}$ ions and at high pressures, the radius-ratio of these two species changes such that it is possible to pack more anions around
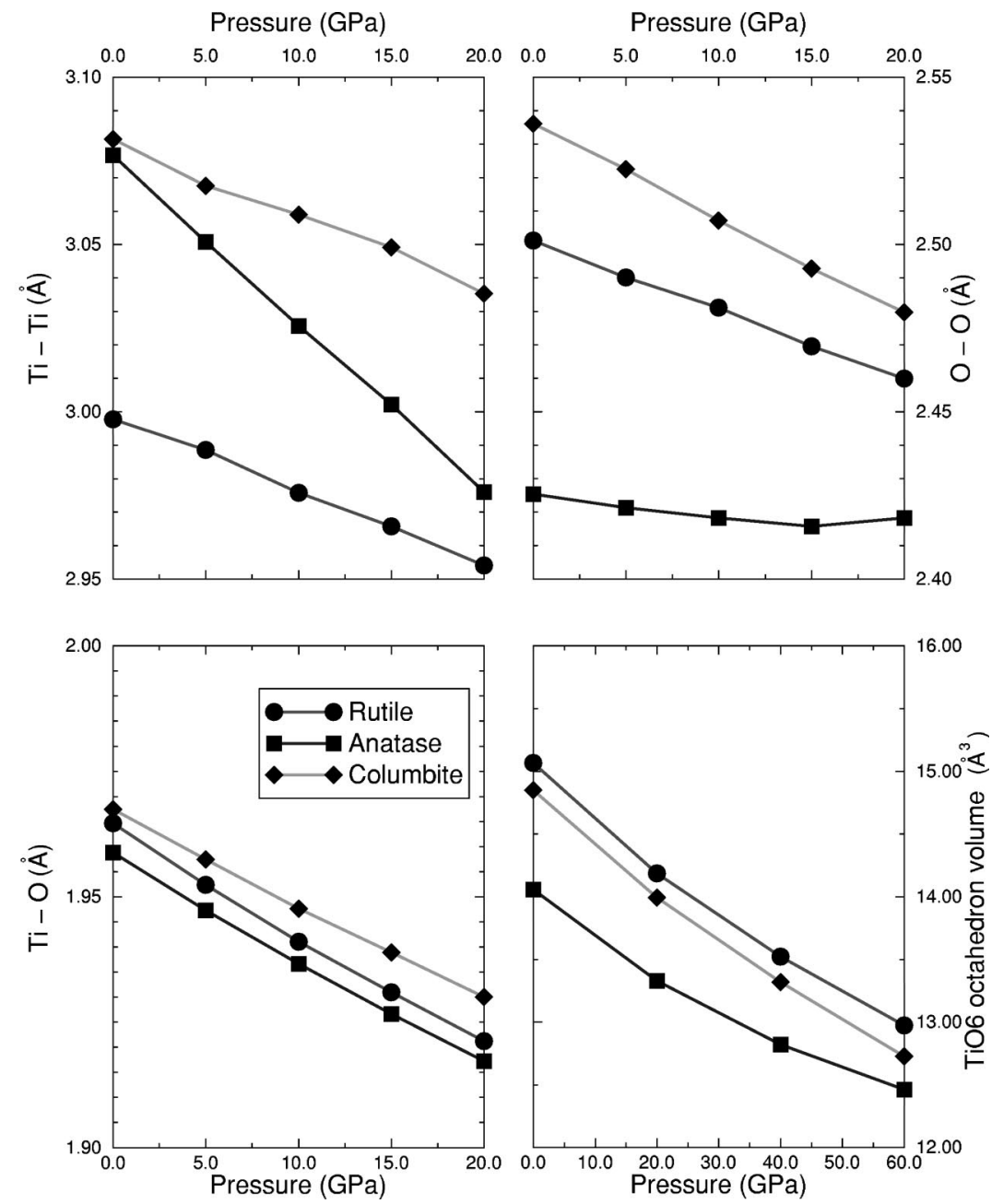

FIG. 9. The change in Ti-Ti, O-O, Ti-O, and $\mathrm{TiO}_{6}$ octahedron volumes with pressure for rutile, anatase, and columbite structured $\mathrm{TiO}_{2}$ computed within the HF approximation. 


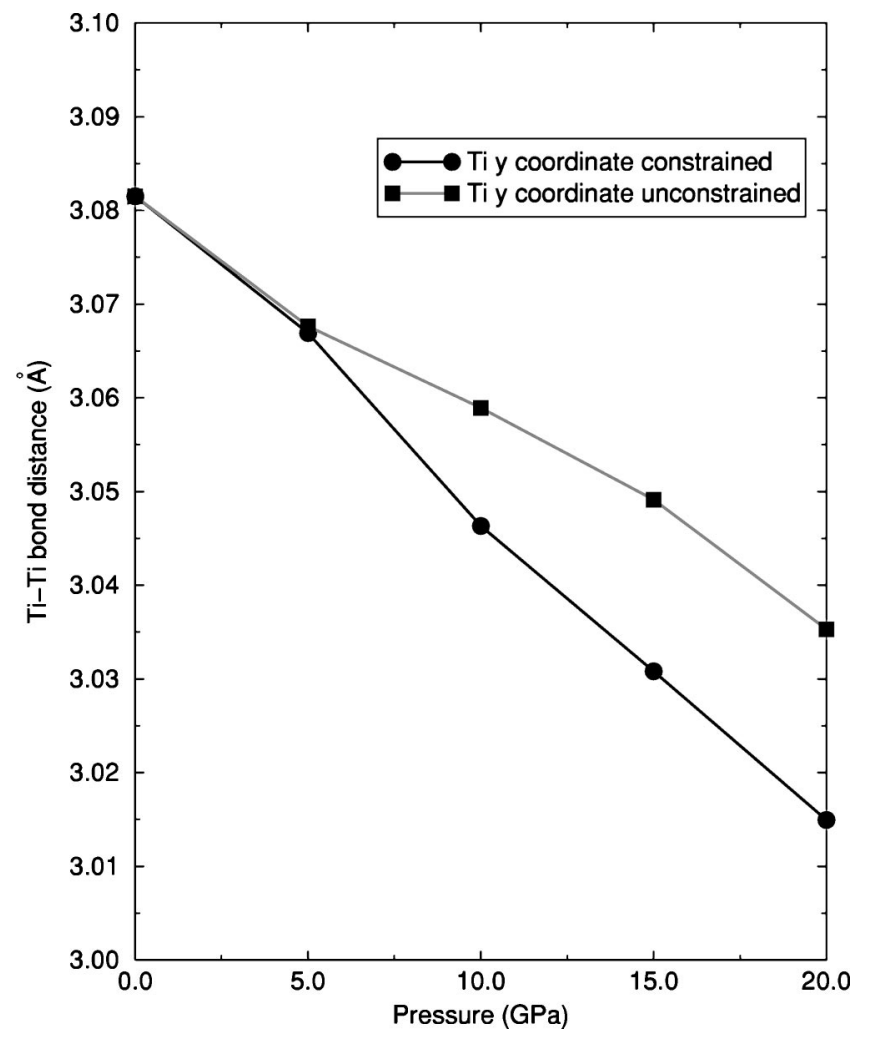

FIG. 10. The nearest Ti-Ti distance in columbite $\mathrm{TiO}_{2}$ with increasing pressure where the Ti $y$ coordinate is fixed (constrained) to its $0-\mathrm{GPa}$ value and when it is free to relax (unconstrained).

each cation hence increasing the Ti-O attraction interaction. This leads to an increase in the coordination of Ti-O ions from six (rutile, anatase, and columbite) to seven (baddeleyite) and then to nine (cotunnite).

\section{CONCLUSIONS}

In the current study, we have investigated the structure, elastic properties, and stability of all the main known $\mathrm{TiO}_{2}$ polymorphs. In addition, we have confirmed the existence of a high-pressure phase recently been observed to be stable above $60 \mathrm{GPa}$.

Particular care has been taken to compute the relative stability of rutile and anatase. The most precise calculations in the present study predict that anatase is more stable than rutile at $0 \mathrm{~K}$, but the energy difference between the two phases is small: between 2 and $10 \mathrm{~kJ} / \mathrm{mol}$. Previous experimental determinations of the stability of rutile and anatase have not conclusively resolved which phase is more stable at low temperatures and, clearly, further experimental work is required.

The computed cell parameters for the experimentally well-studied phases agree to within $2 \%$. The bulk moduli generally agree with experiment to within $10 \%$, although the direction of the error depends on the individual phase: for low-pressure phases, the calculations tend to overestimate the bulk modulus with respect to experiment, whereas for the very high-pressure phases the computed bulk moduli are too low. HF theory tends to predict a higher bulk modulus than

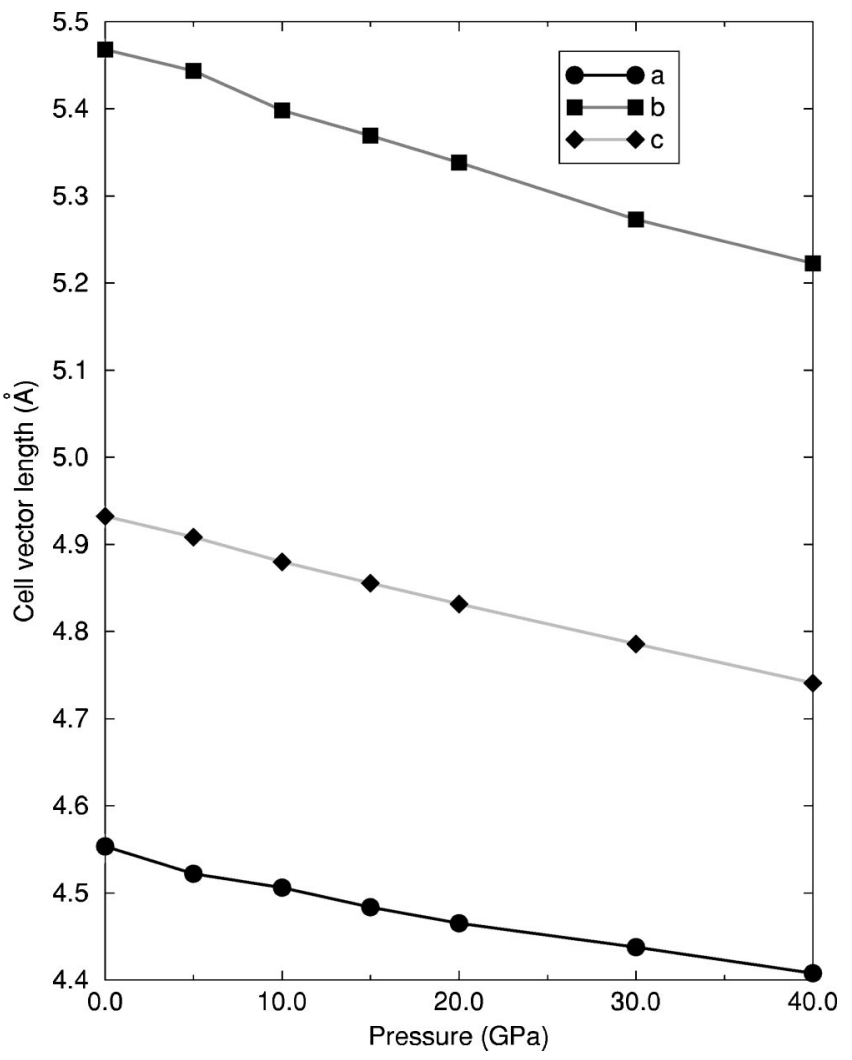

FIG. 11. The $a, b$, and $c$ cell parameters in columbite $\mathrm{TiO}_{2}$ with increasing pressure.

the LDA. A more significant difference in the predicted bulk modulus is seen when comparing that computed using a polynomial fit to the variation of the energy with respect to volume and that obtained by fitting to an equation of state. Here the latter approach is adopted as this is consistent with the approach used in most experimental determinations.

For a number of phases, in particular rutile and anatase, a great deal of insight into their elastic properties can be obtained by considering the local packing of the $\mathrm{Ti}$ and $\mathrm{O}$ ions. For example, the larger, anisotropic compression of the longer cell parameter in rutile and anatase can be rationalized by considering the nature of the packing of the $\mathrm{TiO}_{6}$ octahedral units. The driving force behind the transformation of rutile and anatase to columbite can be understood by considering the changes to the electrostatic interactions that occur as a result of differing cation-cation, anion-anion, and cationanion nearest-neighbor distances. Transformation to the columbite phase allows an increase in the $\mathrm{O}-\mathrm{O}$ and $\mathrm{Ti}-\mathrm{Ti}$ distances particularly due to the extra degree of freedom available for the position of the Ti ions.

The current calculations of the phase transformation of rutile and anatase to columbite correctly predict that anatase undergoes this phase transformation at a lower pressure than rutile. The anatase to columbite transformation is predicted to happen at about $3.5 \mathrm{GPa}$ in reasonable agreement with experiment but the predicted pressure of rutile to columbite transformation is higher than that observed. This is mainly due to the difficulty in extracting the crossover point in the equations of state of the two phases which have very similar 
gradients of the enthalpy versus pressure curves and are very sensitive to the computational parameters used to generate them. We estimate that the error bar in this particular prediction is of the order of $10 \mathrm{GPa}$. The columbite-to-baddeleyite transformation then occurs at $31 \mathrm{GPa}$, and the latter structure transforms to cotunnite at about $63 \mathrm{GPa}$. It is very satisfying to note that all the computed phase transformations are predicted to occur in the same order as is seen experimentally and that the bulk modulus of $\mathrm{TiO}_{2}$ increases with each new phase.

Finally, in this paper, we have demonstrated how the computed properties of bulk $\mathrm{TiO}_{2}$ are influenced by the treatment of exchange and correlation and the numerical approximations (such as the basis set and $k$-space sampling). The results for rutile and anatase illustrate how the treatment of the exchange and correlation affects the computed lattice parameters. Convergence of the basis set, irrespective of its functional form, with respect to computed properties is essential for reliable comparisons between Hartree-Fock and density functional theory results. Reliable structures can be calculated with rather small basis sets but more sophisticated basis sets incorporating $d$-polarization functions on the oxygen ions are necessary to produce accurate lattice parameters for anatase. When the basis set is converged, the HF method and the GGA to DFT theory tend to overestimate cell volumes, whereas the LDA to DFT method underestimates volumes. Other numerical approximations such as the sampling of $k$ space and the truncation of the Coulomb and exchange series have a very small effect on the predicted structures. Modest numerical accuracy is required to converge computed structures, but calculations of the total energies and energy differences between different phases require more sophisticated basis sets with high numerical accuracy.

\section{ACKNOWLEDGMENTS}

J.M. would like to thank the EPSRC for funding part of this work during his Ph.D. studies. He would also like to acknowledge useful discussions with Jose Mirao (University of Evora, Portugal) and Philip Lindan (University of Canterbury, UK). All images of the $\mathrm{TiO}_{2}$ phases were produced using the DL-Visualise software package (Refs. 87 and 88).
${ }^{1}$ V. E. Henrich and A. F. Cox, The Surface Science of Metal Oxides (Cambridge University Press, Cambridge, 1993).

${ }^{2}$ A. Fujishima and K. Honda, Nature (London) 238, 37 (1972).

${ }^{3}$ G. L. Haller and D. E. Resasco, Adv. Catal. 36, 173 (1989).

${ }^{4}$ New Sci. 10, 11 (1998).

${ }^{5}$ L. S. Dubrovinsky, N. A. Dubrovinskaia, V. Swamy, J. Muscat, N. M. Harrison, R. Ahuja, B. Holm, and B. Johansson, Nature (London) 410, 653 (2001).

${ }^{6}$ T. Mitsuhashi and O. J. Kleppa, J. Am. Chem. Soc. 62, 356 (1979).

${ }^{7}$ J. L. Margrave and B. D. Kybett, Tech. Rept. AFMO-TR, 1965, p. 65 .

${ }^{8}$ A. Navrotsky and O. J. Kleppa, J. Am. Ceram. Soc. 50, 626 (1967)

${ }^{9}$ C. N. R. Rao, Can. J. Chem. 39, 498 (1961).

${ }^{10}$ R. A. Robie and D. R. Waldbaum, U. S. Geol. Surv. Bull. 1259, 146 (1966).

${ }^{11}$ A. Fahmi, C. Minot, B. Silvi, and M. Causà, Phys. Rev. B 47, 11717 (1993).

${ }^{12}$ M. Causà and A. Zupan, Chem. Phys. Lett. 220, 145 (1994).

13 V. Milman, Properties of Complex Inorganic Solids (Plenum Press, New York, 1997).

${ }^{14}$ M. Mikami, S. Nakamura, O. Kitao, H. Arakawa, and X. Gonze, Jpn. J. Appl. Phys. 39, 847 (2000).

${ }^{15}$ N. A. Bendeliani, S. V. Popova, and L. F. Vereshchagin, Geochem. Int. 3, 387 (1966).

${ }^{16}$ R. G. McQueen, J. C. Jamieson, and S. P. Marsh, Science 155, 1401 (1967).

${ }^{17}$ J. F. Mammone, S. K. Sharma, and M. Nicol, Solid State Commun. 34, 799 (1980).

${ }^{18}$ J. F. Mammone, M. Nicol, and S. K. Sharma, J. Phys. Chem. Solids 42, 379 (1981)

${ }^{19}$ H. Arashi, J. Phys. Chem. Solids 53, 355 (1992).

${ }^{20}$ L. Liu and T. P. Mernagh, Eur. J. Mineral. 4, 45 (1992).
${ }^{21}$ J. Haines and J. M. Lèger, Physica B 192, 233 (1993).

${ }^{22}$ K. Lagarec and S. Desgreniers, Solid State Commun. 94, 519 (1995).

${ }^{23}$ L. Gerward and J. S. Olsen, J. Appl. Crystallogr. 30, 259 (1997).

${ }^{24}$ J. Staun Olsen, J. Z. Jiang, and L. Gerward, J. Phys. Chem. Solids 60, 229 (1999).

${ }^{25}$ T. Arlt, M. Bermejo, M. A. Blanco, L. Gerward, J. Z. Jiang, J. Staun Olsen, and J. M. Recio, Phys. Rev. B 61, 14414 (2000).

${ }^{26}$ H. Sato, S. Endo, M. Sugiyama, T. Kikegawa, O. Shimomura, and K. Kusaba, Science 251, 786 (1991).

${ }^{27}$ I. E. Grey, C. Li, and I. C. Madsen, Mater. Res. Bull. 23, 743 (1988).

${ }^{28}$ S. Endo, I. Takenaka, and H. Arashi, AIRAPT Conf. Proc. 309, 371 (1979).

${ }^{29}$ Y. Syono, K. Kusaba, M. Kikuchi, and K. Fukuoka, Geophys. Monogr. 39, 385 (1987).

${ }^{30}$ J. K. Dewhurst and J. E. Lowther, Phys. Rev. B 54, 3673 (1996).

${ }^{31}$ J. Muscat, Ph.D. thesis, University of Manchester, Manchester, 1999.

${ }^{32}$ C. R. A. Catlow and R. James, Proc. R. Soc. London, Ser. A 384, 157 (1982).

${ }^{33}$ V. Swamy, J. D. Gale, and L. S. Dubrovinsky, J. Phys. Chem. Solids 62, 887 (2001).

${ }^{34}$ R. Wyckoff, Crystal Structures, 2nd ed. (Interscience, New York, 1964), Vol. 1.

${ }^{35}$ K. V. K. Rao, S. V. N. Naidu, and L. Iyengar, J. Am. Ceram. Soc. 53, 124 (1970).

${ }^{36}$ C. J. Howard, T. M. Sabine, and F. Dickson, Acta Crystallogr., Sect. B: Struct. Sci. 47, 462 (1991).

${ }^{37}$ J. K. Burdett, T. Hughbanks, G. J. Miller, J. W. Richardson, and J. V. Smith, J. Am. Chem. Soc. 109, 3639 (1987).

${ }^{38}$ B.G. Hyde and S. Andersson, Inorganic Crystal Structures (Wiley, New York, 1989).

${ }^{39}$ J. Haines, J. M. Lèger, and O. Schulte, Science 271, 629 (1996). 
${ }^{40}$ R. Dovesi, V. R. Saunders, C. Roetti, M. Causà, N. M. Harrison, R. Orlando, and E. Aprà, CRYSTAL98 User's Manual (University of Turin, Turin, 1996).

${ }^{41}$ M. C. Payne, M. P. Teter, D. C. Allan, T. A. Arias, and J. D. Joannopoulos, CASTEP 4.2 Academic version, licensed under the UKCP-MSI Agreement [Rev. Mod. Phys. 64, 1045 (1992)].

${ }^{42}$ P. A. M. Dirac, Proc. Cambridge Philos. Soc. 26, 376 (1930).

${ }^{43}$ J. P. Perdew and A. Zunger, Phys. Rev. B 23, 5048 (1981).

${ }^{44}$ J. P. Perdew, K. Burke, and M. Ernzerhof, ACS Symp. Ser. 629, 453 (1996).

${ }^{45}$ W. C. Mackrodt, E. A. Simpson, and N. M. Harrison, Surf. Sci. 384, 192 (1997).

${ }^{46}$ J. Muscat, N. M. Harrison, and G. Thornton, Phys. Rev. B 59, 15457 (1999).

${ }^{47}$ http://www.dl.ac.uk/TCS/Software/CRYSTAL/(The CRYSTAL Basis set library, 1998).

${ }^{48}$ J. Muscat, N. M. Harrison, and G. Thornton, Phys. Rev. B 59, 2320 (1999).

${ }^{49}$ J. Muscat and N. M. Harrison, Surf. Sci. 446, 119 (2000).

${ }^{50}$ N. M. Harrison, X.-G. Wang, J. Muscat, and M. Scheffler, Faraday Discuss. 114, 305 (1999).

${ }^{51}$ J. D. Pack and H. J. Monkhorst, Phys. Rev. B 16, 1748 (1977).

${ }^{52}$ C. Pisani, R. Dovesi and C. Roetti, Hartree-Fock Ab Initio Treatment of Crystalline Systems (Springer-Verlag, Berlin, 1988), Vol. 48.

${ }^{53}$ C. Zhu, R. H. Byrd, P. LU, and J. Nocedal, L-BFGS-B - Fortran Subroutines for Large Scale Bound Constrained Optimisation (Dept of Elec. Eng. and Comp. Sci, Northwestern University, Illinois, 1994).

${ }^{54}$ D. Vanderbilt, Phys. Rev. B 41, 7892 (1990).

${ }^{55}$ H. J. Monkhorst and J. D. Pack, Phys. Rev. B 13, 5188 (1976).

${ }^{56}$ N. Funamori, T. Yagi, and T. Uchida, Geophys. Res. Lett. 23, 953 (1996)

${ }^{57}$ B. Silvi, N. Fourati, R. Nada, and C. R. A. Catlow, J. Phys. Chem. Solids 52, 1005 (1991).

${ }^{58}$ P. Reinhardt and B. A. Hess, Phys. Rev. B 50, 12015 (1994).

${ }^{59}$ K. M. Glassford and J. R. Chelikowsky, Phys. Rev. B 46, 1284 (1992).

${ }^{60}$ C. Lee, P. Ghosez, and X. Gonze, Phys. Rev. B 50, 13379 (1994).

${ }^{61}$ M. Ramamoorthy, D. Vanderbilt, and R. D. King-Smith, Phys. Rev. B 49, 16721 (1994).
${ }^{62}$ P. J. D. Lindan, N. M. Harrison, J. M. Holender, M. J. Gillan, and M. C. Payne, Surf. Sci. 364, 431 (1996).

${ }^{63}$ P. Reinhardt, B. A. Hess, and M. Causà, Int. J. Quantum Chem. 58, 297 (1996).

${ }^{64}$ P. J. D. Lindan, N. M. Harrison, M. J. Gillan, and J. A. White, Phys. Rev. B 55, 15919 (1997).

${ }^{65}$ K. Rosciszewski, K. Doll, B. Paulus, P. Fulde, and H. Stoll, Phys. Rev. B 57, 14667 (1998).

${ }^{66}$ S. D. Mo and W. Y. Ching, Phys. Rev. B 51, 13023 (1995).

${ }^{67}$ S. H. Vosko, L. Wilk, and M. Nusair, Can. J. Phys. 58, 1200 (1980).

${ }^{68}$ U. von Barth and L. Hedin, J. Phys. C 5, 1629 (1972).

${ }^{69}$ J. P. Perdew and Y. Wang, Phys. Rev. B 33, 8800 (1986).

${ }^{70}$ J. P. Perdew and Y. Wang, Phys. Rev. B 40, 3399 (1989).

${ }^{71}$ J. P. Perdew and Y. Wang, Phys. Rev. B 45, 13244 (1992).

${ }^{72}$ R. Colle and O. Salvetti, J. Chem. Phys. 93, 534 (1990).

${ }^{73}$ L. C. Wilson and M. Levy, Phys. Rev. B 41, 12930 (1990).

${ }^{74}$ J. P. Perdew, Phys. Rev. B 33, 8822 (1986).

${ }^{75}$ J. P. Perdew, Electronic Structure of Solids 1991 (Akademie Verlag, Berlin, 1991).

${ }^{76}$ A. D. Becke, J. Chem. Phys. 88, 1053 (1988).

${ }^{77}$ C. Lee, W. Yang, and R. G. Parr, Phys. Rev. B 37, 785 (1988).

${ }^{78}$ L. C. Ming and M. H. Manghnani, J. Geophys. Res. 84, 4777 (1979).

${ }^{79}$ M. H. Manghnami, J. Geophys. Res. 74, 4317 (1969).

${ }^{80}$ I. J. Fritz, J. Phys. Chem. Solids 35, 817 (1974).

${ }^{81}$ D. G. Isaak, J. D. Carnes, O. L. Anderson, H. Cynn, and E. Hake, Phys. Chem. Miner. 26, 31 (1998).

${ }^{82}$ V. Swamy and L. S. Dubrovinsky, J. Phys. Chem. Solids 62, 673 (2001).

${ }^{83}$ Y. Kudoh and H. Takeda, Physica B \& C 139-140, 333 (1986).

${ }^{84}$ T. Sekiya, S. Ohta, S. Kamei, M. Hanakawa, and S. Kurita, J. Phys. Chem. Solids 62, 717 (2001).

${ }^{85}$ M. Akaogi, K. Kusaba, J. Susaki, T. Yagi, M. Matsui, T. Kikegawa, H. Yusa and E. Ito, High-Pressure Research: Application to Earth and Planetary Sciences (Terra Scientific Publishing Company/American Geophysical Union, Tokyo, 1993).

${ }^{86} \mathrm{~N}$. Wilson and J. Muscat (unpublished).

${ }^{87}$ B. G. Searle, The DL-Visualise Software package (http:// www.cse.clrc.ac.uk/Activity/DLV/, 2001).

${ }^{88}$ B. G. Searle, Comput. Phys. Commun. 137, 25 (2001). 